\title{
Modèles débit-durée-fréquence d'étiage, concept et usage pour une approche régionale des régimes de basses eaux des bassins hydrographiques de la Loire (France) et du Crisu-Alb (Roumanie) \\ Low flow-duration-frequency models. Concept and use for a regional approach to watershed low-flow regimes in the Loire (France) and Crisu-Alb (Romania) regions
}

\author{
G. Galéa, G. Mercier et M. J. Adler
}

Volume 12, numéro 1, 1999

URI : https://id.erudit.org/iderudit/705345ar

DOI : https://doi.org/10.7202/705345ar

Aller au sommaire du numéro

Éditeur(s)

Université du Québec - INRS-Eau, Terre et Environnement (INRS-ETE)

ISSN

0992-7158 (imprimé)

1718-8598 (numérique)

Découvrir la revue

Citer cet article

Galéa, G., Mercier, G. \& Adler, M. J. (1999). Modèles débit-durée-fréquence d'étiage, concept et usage pour une approche régionale des régimes de basses eaux des bassins hydrographiques de la Loire (France) et du Crisu-Alb (Roumanie). Revue des sciences de l'eau / Journal of Water Science, 12(1), 93-112. https://doi.org/10.7202/705345ar

\section{Résumé de l'article}

La modélisation de synthèse en débit-durée-fréquence des régimes d'étiage observés s'inspire de travaux développés ces dernières années sur les modèles continus (multidurée et multifréquence) de prédétermination des crues (GALEA et PRUDHOMME, 1996). La variabilité des régimes d'étiage du bassin hydrographique de la Loire $\left(\mathrm{S}=117000 \mathrm{~km}^{2}\right)$ est étudiée à partir de deux variables hydrologiques, définies sur une durée $(d)$ continue $(1 \leqslant \mathrm{~d} \leqslant 90 \mathrm{j})$, traduisant deux notions de régime complémentaires : la notion de débit-volume (moyen) minimal (VCNd) annuel et de débit-seuil minimal (QCNd) annuel non dépassé. L'étude statistique multidurée des événements annuels observés en 57 sites sélectionnés, selon la loi log-normale à deux paramètres (écart-type et moyenne), permet de répartir les différents bassins en 4 familles typologiquement homogènes. Pour chacune de ces familles est choisi un bassin versant de référence dont les courbes débit-durée-fréquence normées en débit et durée gagnent alors en représentativité régionale. L'utilisation de ces courbes adimensionnelles est rendue plus aisée, grâce à un formalisme mathématique. La norme de débit retenue est le débit journalier minimal annuel de période moyenne de retour 2 ans. La norme de durée quant à elle est déduite des courbes de tarissement observées et décrites selon l'équation de MAILLET. Ces deux normes (ou encore descripteurs de régime) constituent les seuls paramètres d'entrée des 4 modèles adimensionnels QdF d'étiage. Ces modèles et leur typologie associée, appliqués au bassin hydrographique de la Loire, ont permis de montrer la cohérence des quantiles observés et modélisés en chacun des 57 sites retenus. Une première validation de la méthodologie QdF d'étiage, établie sur le bassin de la Loire, a concerné 12 bassins versants du Crisu-Alb $\left(S=3000 \mathrm{~km}^{2}\right)$ en Roumanie. Cela n'a pas nécessité de nouveaux modèles de référence autres que ceux élaborés pour la Loire. Autrement dit, pour chacun des sites étudiés, la valeur de l'écart-type de la loi ajustée sur les VCNd=1j a permis de choisir la famille hydrologique d'appartenance et donc le modèle QdF d'étiage qui lui est associé. La qualité des modélisations effectuées confirment par ailleurs que les deux descripteurs locaux de régime retenus sont de bons intégrateurs des processus d'écoulement liés aux étiages. D'une manière générale, la démarche a permis d'éprouver la fiabilité de la méthodologie QdF développée en étiage et ces premiers résultats sont encourageants pour l'avenir. Notamment, l'usage opérationnel des modèles QdF d'étiage sur des sites non observés nécessite de poursuivre l'effort de recherche vers une explicitation du critère de choix ainsi que des deux descripteurs locaux du régime de basses eaux. 


\title{
Modèles débit-durée-fréquence d'étiage, concept et usage pour une approche régionale des régimes de basses eaux des bassins hydrographiques de la Loire (France) et du Crisu-Alb (Roumanie)
}

\author{
Low flow-duration-frequency models. Concept and use \\ for a regional approach to watershed low-flow regimes \\ in the Loire (France) and Crisu-Alb (Romania) regions
}

\section{G. GALÉ ${ }^{1 *}$, G. MERCIER ${ }^{1}$ et M.J. ADLER ${ }^{2}$}

Reçu le 11 avril 1997, accepté le 25 mai 1998**.

\section{SUMMARY}

The French Department of the Environment entrusted to the CEMAGREF the realization of a study relating to an ecosystemic approach to the management of the Loire watershed. Over the long term, this approach is designed to establish orientations for the protection and management of the natural aquatic environments of the Loire. To this end, a good knowledge of the variability of the hydrological regimes is necessary. The method presented relates primarily to low flow discharges. In spite of the great heterogeneity in space and time of low flows, a regionalization study of watershed low flow regimes of Loire $\left(S=117000 \mathrm{~km}^{2}\right.$ ) has been attempted on the basis of the same QdF (dischargeduration-frequency) concept developed for floods (GALÉA and PRUDHOMME, 1996). To allow an objective evaluation of developed methodology, we present here the details of the conceptual approach used to model and synthesize the low flow regimes observed, and present the results obtained.

The methodology relates primarily to relatively undisturbed basins with perennial flow. The variability of low flow regimes takes into account two hydrologic variables expressing two notions of complementary regimes: the notion of annual minimal average flow (VCNd), and the annual minimal threshold discharge $(Q C N d)$ below which the flow does not decrease for a continuous duration (d) which varies from one day to 90 days. The statistical study of annual events demonstrates that the Log-Normal law (two parameters) is generally adequate for a given duration d. From the low flow typology observed on 57 selected sites, the statistical study of annual events revealed four homogeneous groups of hydrological low flow regimes. In each of the 4 groups a reference basin is chosen. Its flow-duration-frequency curves normalized to discharge and dnration show a regional representativity and the mathematical formalism yields easy-to-use dimensionless curves. The normative discharge $V C N(2,1)$ is

Cemagref-Lyon, Division Hydrologie Hydraulique, 3 bis, quai Chauveau, 69336 Lyon cedex 09, France.

INMH, Soseaua Bucarest-Ploiesti 97 Secteur 1, 71552 Bucarest, Roumanie.

* Correspondance.

** Les commentaires seront reçus jusqu'au 30 septembre 1999. 
the minimum annual daily flow for a two year return period. The normative duration of flow ( $D e$ ) of a basin results from the recession curves described by MAILLET's equation. In each group, a large diversity of low flow regimes exists, as indicated by the two local descriptors ( $D e$ and $V C N(2,1)$ ), which are the only input parameters for the low flow QdF models. Concerning the Loire watershed, these 4 models and their associated typology characterize the large diversity in space and time of low flows observed for the 57 studied cases.

A first validation of the low flow QdF methodology concerned 12 basins of Crisu-Alb watershed, in Romania. It was not necessary to establish new reference models for the regionalization of low flows for these 12 studied cases in the Crisu-Alb watershed. The quality of the transposed models confirms, on another hand, that the two local descriptors of the low flow regime are good integrators of flow processes in relation with low flows. The initial results from the synthetic low flow models are promising. Use of these models on ungauged basins will require additional research to explain the model's choice criterion and the two local descriptors of the low flow regime.

Key-words: low flow, statistical hydrology, synthetic models (low flow-durationfrequency), regionalization.

La modélisation de synthèse en débit-durée-fréquence des régimes d'étiage observés s'inspire de travaux développés ces dernières années sur les modèles continus (multidurée et multifréquence) de prédétermination des crues (GALÉA et PRUDHOMME, 1996). La variabilité des régimes d'étiage du bassin hydrographique de la Loire $\left(S=117000 \mathrm{~km}^{2}\right)$ est étudiée à partir de deux variables hydrologiques, définies sur une durée (d) continue $(1 \mathrm{j} \leq \mathrm{d} \leq 90 \mathrm{j})$, traduisant deux notions de régime complémentaires : la notion de débit-volume (moyen) minimal (VCNd) annuel et de débit-seuil minimal (QCNd) annuel non dépassé. L'étude statistique multidurée des événements annuels observés en 57 sites sélectionnés, selon la loi log-normale à deux paramètres (écart-type et moyenne), permet de répartir les différents bassins en $\mathbf{4}$ familles typologiquement homogènes. Pour chacune de ces familles est choisi un bassin versant de référence dont les courbes débit-durée-fréquence normées en débit et durée gagnent alors en représentativité régionale. L'utilisation de ces courbes adimensionnelles est rendue plus aisée, grâce à un formalisme mathématique. La norme de débit retenue est le débit journalier minimal annuel de période moyenne de retour 2 ans. La norme de durée quant à elle est déduite des courbes de tarissement observées et décrites selon l'équation de MAILLET. Ces deux normes (ou encore descripteurs de régime) constituent les seuls paramètres d'entrée des 4 modèles adimensionnels QdF d'étiage. Ces modèles et leur typologie associée, appliqués au bassin hydrographique de la Loire, ont permis de montrer la cohérence des quantiles observés et modélisés en chacun des $\mathbf{5 7}$ sites retenus. Une première validation de la méthodologie QdF d'étiage, établie sur le bassin de la Loire, a concerné 12 bassins versants du Crisu-Alb $\left(S=3000\right.$ km$\left.^{2}\right)$ en Roumanie. Cela n'a pas nécessité de nouveaux modèles de référence autres que ceux élaborés pour la Loire. Autrement dit, pour chacun des sites étudiés, la valeur de l'écart-type de la loi ajustée sur les VCNd $=1 \mathrm{j}$ a permis de choisir la famille hydrologique d'appartenance et donc le modèle QdF d'étiage qui lui est associé. La qualité des modélisations effectuées confirment par ailleurs que les deux descripteurs locaux de régime retenus sont de bons intégrateurs des processus d'écoulement liés aux étiages. D'une manière générale, la démarche a permis d'éprouver la fiabilité de la méthodologie QdF développée en étiage et ces premiers résultats sont encourageants pour l'avenir. Notamment, l'usage opérationnel des modèles QdF d'étiage sur des sites non observés nécessite de poursuivre l'effort de recherche vers une explicitation du critère de choix ainsi que des deux descripteurs locaux du régime de basses eaux.

Mots clés : débits d'étiage, hydrologie statistique, modèles synthétiques (débitdurée-fréquence), régionalisation. 


\section{1 - INTRODUCTION}

Le ministère de l'Environnement a confié au Cemagref la réalisation d'une étude portant sur une approche écosystémique du bassin de la Loire (Cemagref, CNRS, 1993). Elle a pour objectif de jeter les bases d'un système de référence quantitatif régionalisé des écosystèmes aquatiques selon leur appartenance typologique (hydroécorégions). Elle devrait permettre à terme de définir des orientations de protection et de gestion des milieux aquatiques naturels du bassin de la Loire.

La gestion rationnelle de la ressource en eau, à l'échelle des bassins versants en général et des écosystèmes aquatiques en particulier, nécessite une bonne connaissance de la variabilité des régimes hydrologiques en conditions fréquentes ainsi qu'en période de crise liée aux événements de crue (SOURISSEAU et GALÉA, 1996) et d'étiage plus rares. L'étude de la variabilité des régimes hydrologiques d'étiage (MERCIER et GALÉA, 1996), dans le cadre des " orientations de protection et de gestion des milieux aquatiques naturels du bassin versant de la Loire ", a nécessité le développement d'une méthodologie QdF en étiage dont les premiers tests ont été initiés par KAABI (1994). Comme la méthodologie QdF en crue (GALÉA et PRUDHOMME, 1994, 1996), dont elle s'inspire, elle résulte d'une approche des différentes typologies de régime d'étiage observées et de l'identification de deux descripteurs locaux explicites de la variabilité temporelle des étiages.

\section{2 - ANALYSE PROBABILISTE DES CHRONIQUES DE DÉBIT JOURNALIER}

Sur les 76 bassins versants retenus initialement (SOURISSEAU et GALÉA, 1996) de superficie variant de quelques dizaines de $\mathrm{km} 2$ à environ $3000 \mathrm{~km}^{2}$, seuls 57 bassins ont fait l'objet d'une étude fréquentielle des étiages (MERCIER et GALÉA, 1996) à partir des chroniques de débit journalier. Les 19 bassins non retenus l'ont été pour diverses raisons : cours d'eau asséché chaque année, forte anthropisation du régime (pompage, dérivation, rejet d'eau résiduaire important, etc.), stations peu fiables en basses eaux, etc. Pour les 57 bassins sélectionnés, la caractérisation du régime d'étiage (figure 1) s'effectue à partir de deux variables de débit caractéristique (GALÉA et PRUDHOMME, 1996) : le VCNd, débit moyen (Volume) Caractéristique sur une durée continue $\mathrm{d}$, miNimal dans la saison (ici l'année) et le QCNd, débit seuil (Q) Caractéristique continûment non dépassé sur la durée d, miNimal dans la saison (ici l'année).

La loi Log-Normale à deux paramètres, d'utilisation fréquente en étiage (KAABI, 1994 ; OBERLIN, LEDUC et GAZULL, 1989 ; CTGREF, 1979) présente pour les différents échantillons constitués sur les différents sites (ex. : bassin du Gartempe à Bessines, figure 2) une adéquation acceptable, consolidée par l'approche multidurée $(1 j \leq d \leq 90 \jmath)^{1}$ des caractéristiques de débit (VOGEL et KROLL, 1991). Les dis-

1 Les quantiles humides d'étiage à 90 jours représentent déjà des moyennes eaux (ills sont donnés pour intormation). 


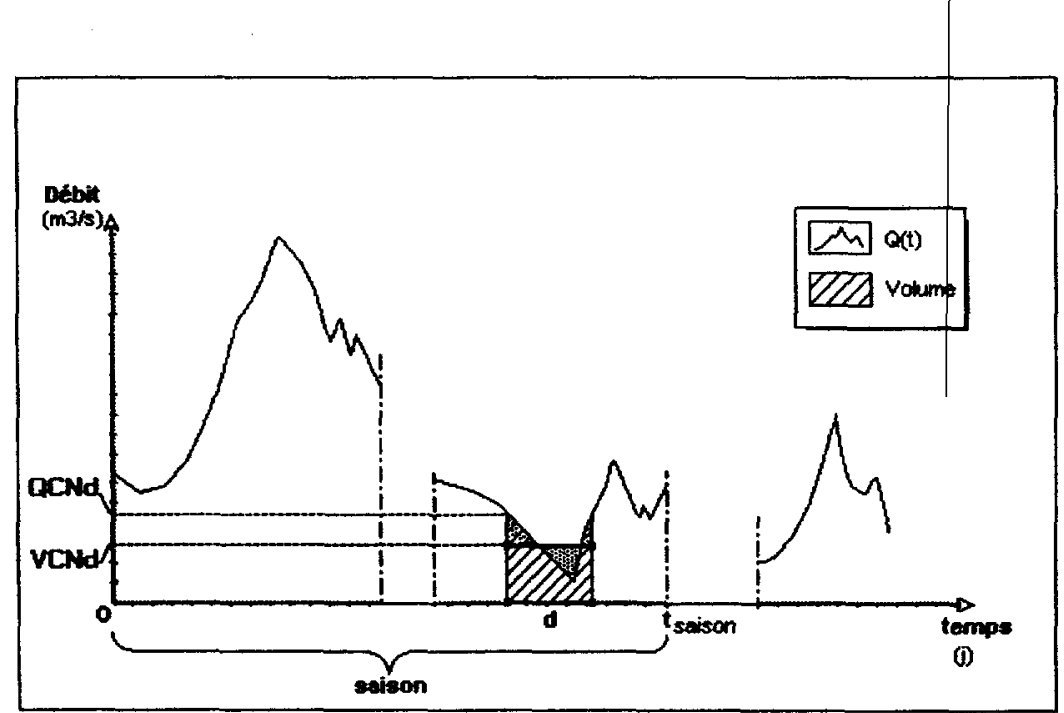

Figure 1 Variables caractéristiques d'étiage.

Characteristics variables of the low flow regime.

tributions expérimentales et théoriques concernant les VCNd (ou Q) se visualisent dans le graphe de la figure 3 :

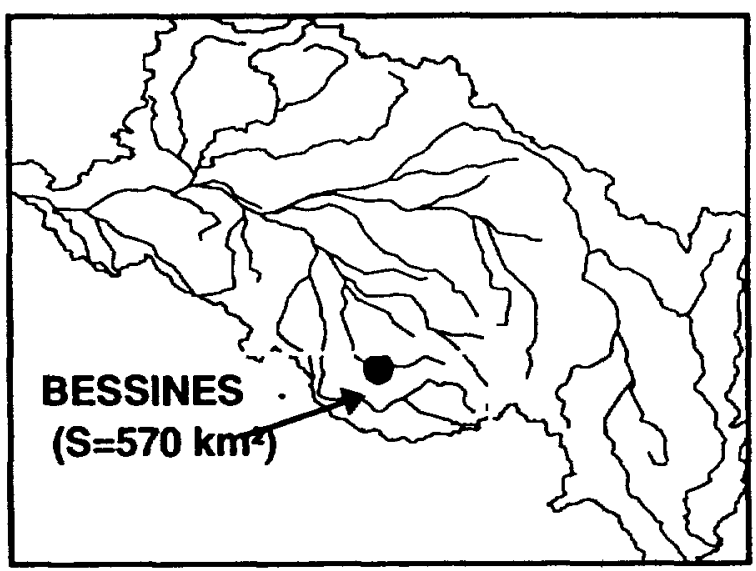

Figure 2 Localisation.

Location.

L'échantillon des VCNd est classé par ordre croissant et un indice de rang i est affecté à chaque valeur. Ainsi la valeur la plus faible se voit attribuer le rang $i=1$ et la valeur la plus forte le rang $i=n$ (échantillon de $n$ valeurs). 


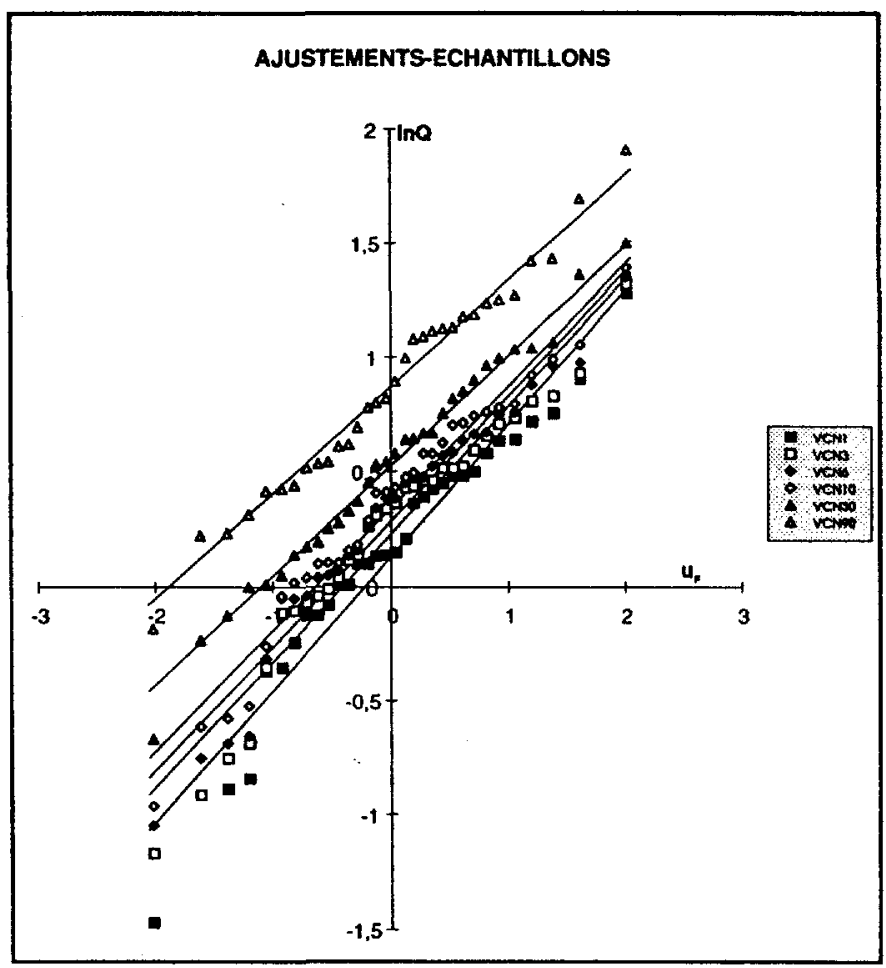

Figure 3 Bassin versant du Gartempe à Bessines.

Gartempe's basin at Bessines.

La fréquence empirique $F_{i}$ est déduite de l'expression : $F_{i}=\frac{i-0,3}{n+0,4}$, dite de Chegodayev (LUBES et al., 1994). Selon la valeur de $F_{i}$, on parlera de quantile fort ou de quantile faible.

Quantile humide (ou fort) : $F_{i}>0,5 \quad u_{F}>0 \quad T=\frac{1}{1-F_{i}}$

Quantile sec (ou faible) : $\quad F_{i}<0,5 \quad U_{F}<0 \quad T=\frac{1}{F_{i}}$

La loi de distribution théorique a pour équation :

$$
\ln (\operatorname{VCN}(T, d))=m(d)+s(d) \cdot u_{F}
$$

où : $m(d)$ : moyenne des logarithmes népériens des VCNd échantillonnés ;

$s(d)$ : écart-type sur les logarithmes népériens des VCNd échantillonnés ;

$\mathrm{u}_{\mathrm{F}}$ : variable réduite de Gauss ;

$T$ : période moyenne de retour (an).

$$
m(d)=\frac{\sum \ln (V C N d)}{n} \quad \text { et } \quad s(d)=\sqrt{\frac{\sum(\ln (V C N d)-m(d))^{2}}{n-1}}
$$


La distinction entre quantiles secs et quantiles humides de période moyenne de retour $(T)$ donnée (liée à $U_{F}$ ) est une forme de représentation commode pour le gestionnaire de bassins versants. Les quantiles $Q(T, d)$ correspondants se déduisent de la loi de distribution théorique précédente, avec les valeurs de $s(d)$ et $m$ (d) du tableau 1.

Tableau 1 de la figure 3.

Table 1, figure 3.

\begin{tabular}{|c|c|c|}
\hline \multicolumn{3}{|c|}{$\begin{array}{c}\text { Code hydrologique : L. 5101810 } \\
\text { Période d'observation : 1959-1993 }\end{array}$} \\
\hline \multicolumn{3}{|c|}{ Ajustement de la loi Log-normale } \\
\hline Durée d(j) & $\mathbf{s ( d )}$ & $\mathbf{m}(\mathbf{d})$ \\
\hline 1 & 0,583 & 0,126 \\
3 & 0,557 & 0,228 \\
6 & 0,547 & 0,281 \\
10 & 0,533 & 0,340 \\
30 & 0,480 & 0,525 \\
90 & 0,464 & 0,870 \\
\hline
\end{tabular}

Tableau 2 Bassin du Gartempe à Bessines - quantiles d'étiage des observations.

Table 2 Gartempe's basin at Bessines - low flows quantiles from observations.

\begin{tabular}{|c|c|c|c|c|c|c|c|c|c|}
\hline \multirow{6}{*}{$\begin{array}{c}\text { Durée } \\
\text { continue } \\
d(j)\end{array}$} & \multicolumn{9}{|c|}{$\operatorname{VCN}(T, d)\left(m^{3} / s\right)$} \\
\hline & \multicolumn{5}{|c|}{ Quantiles secs } & \multicolumn{4}{|c|}{ Quantiles humides } \\
\hline & \multicolumn{9}{|c|}{$T$ (an) } \\
\hline & 50 & 20 & 10 & 5 & 2 & 5 & 10 & 20 & 50 \\
\hline & \multicolumn{9}{|c|}{$U_{F}$} \\
\hline & $-2,055$ & $-1,64$ & $-1,28$ & $-0,84$ & 0 & 0,84 & 1,28 & 1,64 & 2,055 \\
\hline 1 & 0,342 & 0,436 & 0,538 & 0,695 & 1,134 & 1,851 & 2,392 & 2,951 & 3,759 \\
\hline 3 & 0,400 & 0,504 & 0,616 & 0,787 & 1,256 & 2,005 & 2,562 & 3,131 & 3,946 \\
\hline 6 & 0,430 & 0,540 & 0,658 & 0,837 & 1,324 & 2,097 & 2,668 & 3,248 & 4,076 \\
\hline 10 & 0,470 & 0,586 & 0,710 & 0,898 & 1,405 & 2,198 & 2,779 & 3,367 & 4,201 \\
\hline 30 & 0,630 & 0,769 & 0,914 & 1,130 & 1,690 & 2,530 & 3,125 & 3,714 & 4,533 \\
\hline 90 & 0,920 & $\uparrow, 115$ & 1,318 & 1,616 & 2,387 & 3,525 & 4,323 & 5,109 & 6,194 \\
\hline
\end{tabular}

La loi Log-Normale, qui présente en général une bonne adéquation aux échantillons de VCNd et QCNd constitués $(1 j \leq d \leq 90 j$ ) aux 57 sites retenus, peut être aussi utilisée dans le cas de cours d'eau non pérennes (loi tronquée de quelques valeurs nulles). Cette loi simple peut donc constituer le premier support d'une démarche exploratoire, dite $\mathrm{QdF}$ étiage, de régionalisation des régimes d'étiage du bassin hydrographique de la Loire. 


\section{3 - BASSINS VERSANTS ET ABAQUES DE RÉFÉRENCE, TRANSFERTS DE QUANTILES}

\subsection{Typologie des régimes d'étiage}

L'étude fréquentielle des étiages aux 57 sites a permis, à partir de la pente (écart-type) des ajustements réalisés pour les VCNd ( $d=1$ jour, durée la plus performante pour la norme de débit, §3.3), d'identifier empiriquement quatre classes d'amplitude variable ou encore appelées familles hydrologiques. Chacune d'elle est représentée par un bassin versant de référence (cf. ci-dessous) dont la situation (figure 11) est indiquée dans le contexte typologique des régimes d'étiage étudiés. Autrement dit, l'écart-type de la loi ajustée sur l'échantillon de débit journalier minimal annuel permet d'identifier (critère de choix) la famille hydrologique représentative du bassin versant étudié. Selon ce critère, on peut donner la tendance générale des régimes hydrologiques d'étiage des bassins versants appartenant à chaque famille (ou modèle) hydrologique.

- les bassins versants des familles de MENETREOL $(s(d=1 j) \leq 0,4)$ et de SAINT-FLORET $(0,4<s(d=1 j) \leq 0,54)$ présentent une faible différenciation (pente faible) des quantiles journaliers secs et humides. Le régime d'étiage est dit soutenu.

- pour les bassins versants des familles de VAUBARLET $(0,54<\mathrm{s}(\mathrm{d}=1 \mathrm{j}) \leq 0,8)$ et de FORCE $(0,8<s(d=1 j) \leq 1)$ la pente est forte, le régime d'étiage est peu soutenu.

\begin{tabular}{r|l} 
& $\begin{array}{l}\text { s (écart-type) } \\
\text { Régime peu soutenu }\end{array}$ \\
\hline 1 & Modèle de FORCE \\
\hline 0,8 & Modèle de VAUBARLET \\
\hline 0,54 & Modèle de SAINT-FLORET \\
\hline 0,4 & Modèle de MENETREOL \\
& Régime bien soutenu
\end{tabular}

Les 19 bassins versants pour lesquels le facteur anthropique s'est révélé trop important et qui n'ont pas été retenus présentent généralement des pentes d'ajustements supérieures à celles de FORCE $(s(d=1 j)>1)$. Ce fait peut être expliqué par une demande en eau d'autant plus importante que la ressource est faible. Ainsi, les prélèvements d'eau pour l'irrigation en particulier accentuent la différence entre les débits des années sèches (forts prélèvements) et ceux des années humides (prélèvements moindres), d'où des pentes d'ajustements exagérément fortes. 
Notons également, que les écart-types (relatifs à $d=1 \mathrm{j}$ ) de certains bassins (au nombre de 6 sur les 57 retenus), obtenus pour des échantillons tronqués des valeurs de débit nulles, ne sont en aucun cas représentatifs du régime d'étiage et ne permettent donc pas d'identifier la famille hydrologique d'appartenance de chacun de ces 6 bassins versants. Pour l'instant, en l'absence de critère de choix spécifique, ces bassins versants non pérennes ont été répartis entre les 4 familles selon le modèle d'étiage présentant la meilleure adéquation.

\subsection{Descripteurs locaux du régime d'étiage}

- Le descripteur de débit est le VCN(T = 2 ans, $d=1$ jour $)$, quantile de débitVolume (moyen) journalier Caractéristique sur une durée continue $d$, miNimal dans la saison (ici l'année), de période moyenne de retour (T) 2 ans. D'autres durées ( $d \geq 1 \mathrm{j}$ ) ont été testées (MERCIER et GALÉA, 1996) mais se sont révélées peu performantes pour la norme de débit (cf. § 3.3).

- Le descripteur de durée est la durée caractéristique d'étiage (De) du bassin versant qui renseigne sur la capacité (au sens qualitatif) de l'aquifère à soutenir le régime de basses eaux. De est déduite de l'analyse des courbes de tarissement décrites selon l'équation de MAILLET :

$$
Q(t)=Q\left(t_{0}\right) \cdot \exp \left(-\alpha \cdot\left(t-t_{0}\right)\right)
$$

où : $Q(t)$ est le débit à l'instant $t$,

$Q\left(t_{0}\right)$ est le débit à l'instant $t_{0}$,

$\alpha$ une constante qui correspond à l'inverse d'un temps.

À partir de la chronique de débit journalier sont sélectionnés des épisodes (i) de tarissement, pour chacun desquels est calculé $\alpha_{i}$ par la relation :

$$
\alpha_{i}=\frac{1}{\left(t-t_{0}\right)} \cdot \ln \left(\frac{Q\left(t_{0}\right)}{Q(t)}\right)
$$

Le graphe $\alpha_{i}=f$ (événements) de la figure 4 montre, pour deux bassins versants observés (l'Allier à Monistrol d'allier et l'Auxance au Pont de Rochecourbe), deux nuages de points distincts d'où la durée caractéristique d'étiage ( $D e$ ) peut être respectivement déduite et telle que $\mathrm{De}=1 / \alpha_{\text {médian, }}$ (ex. : du Gartempe à Bessines, figure 5).

Compte tenu de la dispersion de $\alpha_{i}$, De est un descripteur relativement peu sensible (cf. $\S 7$ ) dont l'ordre de grandeur ( $\mathrm{De}=1 / \alpha_{\text {médian }}$ ) est cependant satisfaisant pour décrire les régimes d'étiage (cf. $\S 5$ et 6 ) identifiés selon les 4 typologies.

\subsection{Abaques de référence}

À partir des courbes QdF (déduites de l'analyse probabiliste, § 2) et des descripteurs locaux de régime, respectifs à chaque bassin versant de référence, peuvent être déduits les abaques adimensionnels de chaque famille hydrologique d'étiage. À titre d'exemple, aux figures 6 et 7 sont respectivement représentés les abaques (en VCNd) en années sèches et en années humides du bassin de référence de VAUBARLET $\left(\operatorname{VCN}(2,1)=0,313 \mathrm{~m}^{3} / \mathrm{s}, D e=14,9 \mathrm{j}\right)$. Pour toute plage de $d / \mathrm{De}$, sont donnés les paramètres $a(T)$ et $b(T)$ des droites (segments), d'équation générale ci-après, où $Q(T, d)$ représente indifféremment $V C N(T, d)$ et $Q C N(T, d)$. 


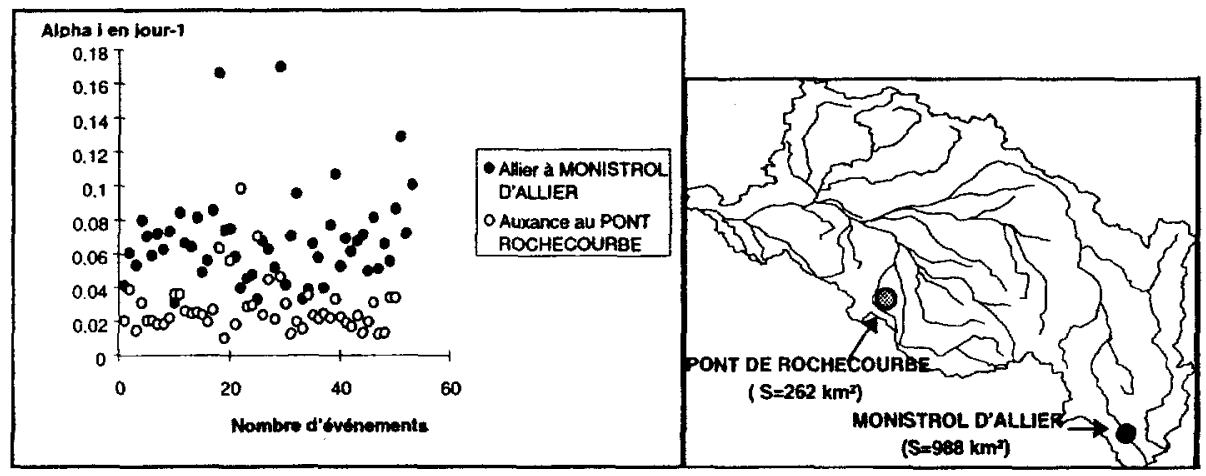

Figure 4 Différenciation des De en deux sites observés.

De differenciation in two jauged basins.

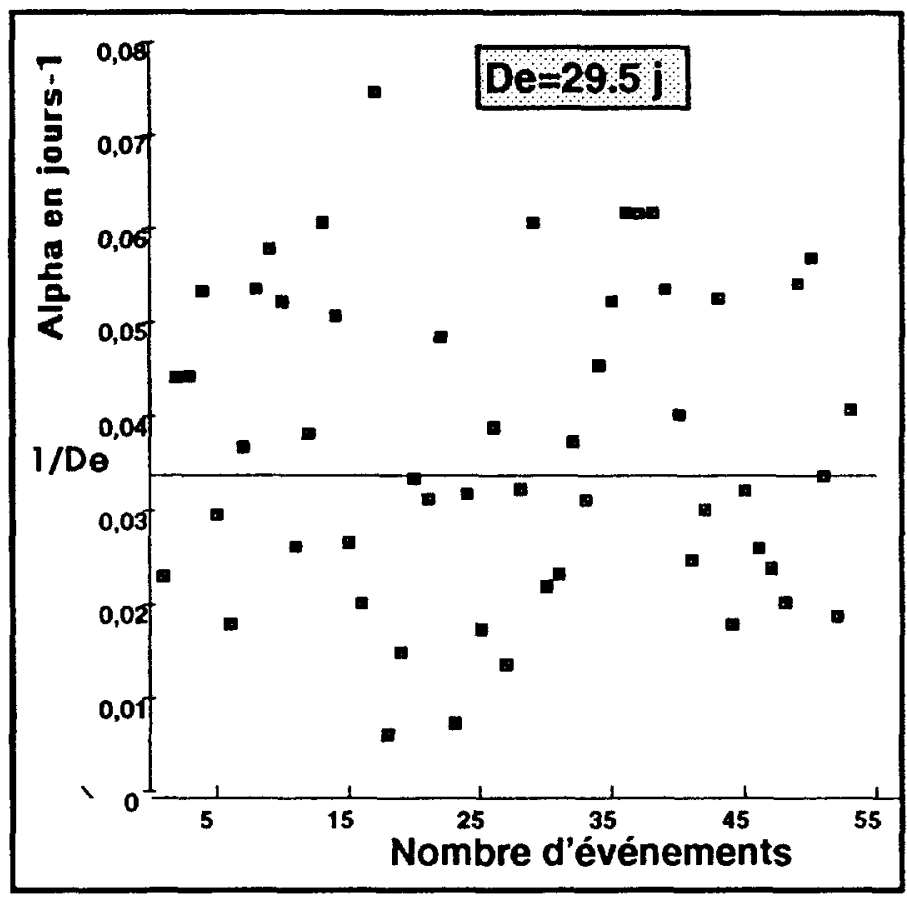

Figure 5 Gartempe à Bessines.

Bessines's basin : De estimation.

$$
\frac{Q(T, d)}{\operatorname{VCN}(T=2 \text { ans, } d=1 \text { jour })}=a(T) \cdot \frac{d}{D e}+b(T)
$$




\begin{tabular}{|c|c|c|c|c|}
\hline \multirow{2}{*}{$T$ (an) } & \multicolumn{2}{|c|}{$d / D e \leq 1$} & \multicolumn{2}{c|}{$1<d / D e \leq 9$} \\
\cline { 2 - 5 } & $a(T)$ & $b(T)$ & $a(T)$ & $b(T)$ \\
\hline 50 & 0,2398 & 0,2189 & 0,0454 & 0,4241 \\
20 & 0,2832 & 0,2959 & 0,0633 & 0,5246 \\
10 & 0,3247 & 0,3840 & 0,0841 & 0,6303 \\
5 & 0,3794 & 0,5272 & 0,1182 & 0,7878 \\
2 & 0,4864 & 0,9622 & 0,2233 & 1,2014 \\
\hline
\end{tabular}

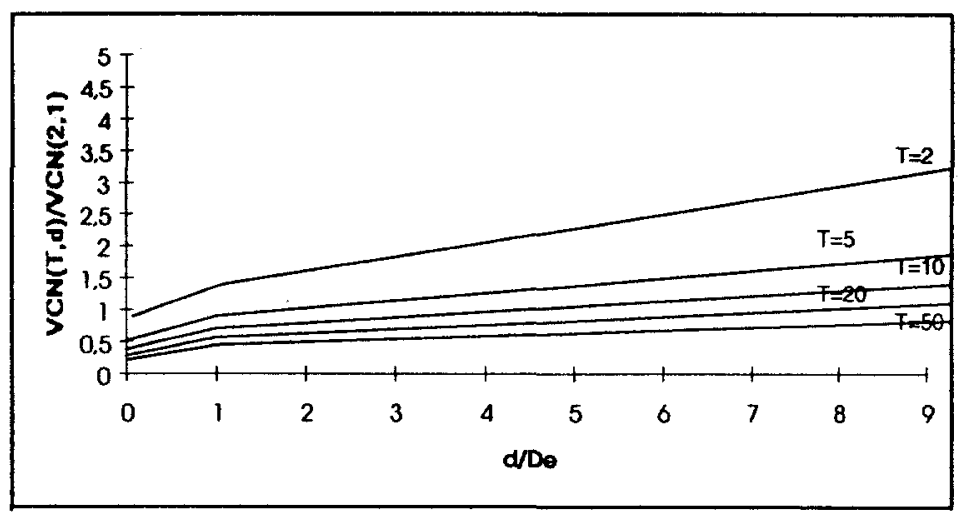

Figure 6 Abaques années sèches Vaubarlet.

Standardized curves of Vaubarlet for dry years

\begin{tabular}{|c|c|c|c|c|}
\hline \multirow{2}{*}{$T(a n)$} & \multicolumn{2}{|c|}{$d / D e \leq 2,7$} & \multicolumn{2}{c|}{$2,7<d / D e \leq 8,72$} \\
\cline { 2 - 5 } & $\mathbf{a}(\mathbf{T})$ & $\mathbf{b}(\mathbf{T})$ & $\mathbf{a}(\mathbf{T})$ & $\mathbf{b}(\mathbf{T})$ \\
\hline $\mathbf{5}$ & $\mathbf{0 , 4 1 9 8}$ & $\mathbf{1 , 8 0 5 4}$ & 0,4198 & 1,8054 \\
10 & 0,5478 & 2,3591 & 0,5478 & 2,3591 \\
20 & $\mathbf{0 , 5 3 2 1}$ & 3,0633 & 0,8455 & 2,1962 \\
50 & 0,5719 & 4,0363 & 1,1738 & 2,4502 \\
\hline
\end{tabular}

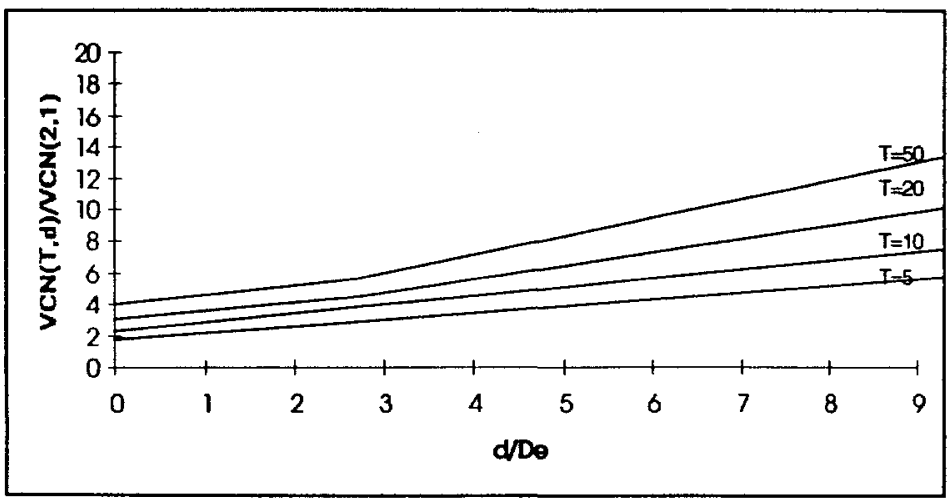

Figure 7 Abaques années humides Vaubarlet.

Standardized curves of Vaubarlet for humid years. 


\subsection{Transfert de quantiles d'étiage : ex. du bassin du Gartempe à Bessines}

Le bassin du Gartempe à Bessines, de descripteurs locaux $\mathrm{De}=29,5$ (figure 5) et $\operatorname{VCN}(2,1)=1,134 \mathrm{~m}^{3} / \mathrm{s}$ (tableau 2), appartient selon le critère typologique défini au $\S 3.1(s(d=1 j)=0,583$, tableau 1$)$ à la famille hydrologique de Vaubarlet. À partir des abaques des figures 6 et 7 et des descripteurs locaux précédemment définis, il est possible d'y transférer des quantiles d'étiage. Le tableau 3 et la figure 8 permettent la comparaison des quantiles observés (tableau 3 : première ligne de durée donnée) et transférés (tableau 3 : deuxième ligne pour une même durée).

Tableau 3 Bassin versant du Gartempe à Bessines $\left(S=570 \mathrm{~km}^{2}\right)$ - quantiles observés et transférés.

Table 3 Gartempe's basin at Bessines (S $\left.=570 \mathrm{~km}^{2}\right)$ - observed and transfered quantiles.

\begin{tabular}{|c|c|c|c|c|c|c|c|c|c|}
\hline \multirow{6}{*}{$\begin{array}{c}\text { Durée } \\
\text { continue } \\
d(j)\end{array}$} & \multicolumn{9}{|c|}{$\operatorname{VCN}(T, d)\left(m^{3} / s\right)$} \\
\hline & \multicolumn{5}{|c|}{ Quantiles secs } & \multicolumn{4}{|c|}{ Quantiles humides } \\
\hline & \multicolumn{9}{|c|}{$T$ (an) } \\
\hline & 50 & 20 & 10 & 5 & 2 & 5 & 10 & 20 & 50 \\
\hline & \multicolumn{9}{|c|}{$\mathbf{U}_{\mathbf{F}}$} \\
\hline & $-2,055$ & $-1,64$ & $-1,28$ & $-0,84$ & 0 & 0,84 & 1,28 & 1,64 & 2,055 \\
\hline 1 & $\begin{array}{l}0,342 \\
0,257\end{array}$ & $\begin{array}{l}0,436 \\
0,346\end{array}$ & $\begin{array}{l}0,538 \\
0,448\end{array}$ & $\begin{array}{l}0,695 \\
0,612\end{array}$ & $\begin{array}{l}1,134 \\
1,110\end{array}$ & $\begin{array}{l}1,851 \\
2,063\end{array}$ & $\begin{array}{l}2,392 \\
2,696\end{array}$ & $\begin{array}{l}2,951 \\
3,494\end{array}$ & $\begin{array}{l}3,759 \\
4,599\end{array}$ \\
\hline 3 & $\begin{array}{l}0,400 \\
0,276\end{array}$ & $\begin{array}{l}0,504 \\
0,368\end{array}$ & $\begin{array}{l}0,616 \\
0,473\end{array}$ & $\begin{array}{l}0,787 \\
0,642\end{array}$ & $\begin{array}{l}1,256 \\
1,147\end{array}$ & $\begin{array}{l}2,005 \\
2,096\end{array}$ & $\begin{array}{l}2,562 \\
2,738\end{array}$ & $\begin{array}{l}3,131 \\
3,535\end{array}$ & $\begin{array}{l}3,946 \\
4,643\end{array}$ \\
\hline 6 & $\begin{array}{l}0,430 \\
0,304\end{array}$ & $\begin{array}{l}0,540 \\
0,401\end{array}$ & $\begin{array}{l}0,658 \\
0,510\end{array}$ & $\begin{array}{l}0,837 \\
0,685\end{array}$ & $\begin{array}{l}1,324 \\
1,203\end{array}$ & $\begin{array}{l}2,097 \\
2,144\end{array}$ & $\begin{array}{l}2,668 \\
2,802\end{array}$ & $\begin{array}{l}3,248 \\
3,596\end{array}$ & $\begin{array}{l}4,076 \\
4,709\end{array}$ \\
\hline 10 & $\begin{array}{l}0,470 \\
0,340\end{array}$ & $\begin{array}{l}0,586 \\
0,444\end{array}$ & $\begin{array}{l}0,710 \\
0,560\end{array}$ & $\begin{array}{l}0,898 \\
0,744\end{array}$ & $\begin{array}{l}1,405 \\
1,278\end{array}$ & $\begin{array}{l}2,198 \\
2,209\end{array}$ & $\begin{array}{l}2,779 \\
2,886\end{array}$ & $\begin{array}{l}3,367 \\
3,678\end{array}$ & $\begin{array}{l}4,201 \\
4,797\end{array}$ \\
\hline 30 & $\begin{array}{l}0,630 \\
0,533\end{array}$ & $\begin{array}{l}0,769 \\
0,668\end{array}$ & $\begin{array}{l}0,914 \\
0,812\end{array}$ & $\begin{array}{l}1,130 \\
1,030\end{array}$ & $\begin{array}{l}1,690 \\
1,620\end{array}$ & $\begin{array}{l}2,530 \\
2,531\end{array}$ & $\begin{array}{l}3,125 \\
3,307\end{array}$ & $\begin{array}{l}3,714 \\
4,087\end{array}$ & $\begin{array}{l}4,533 \\
5,237\end{array}$ \\
\hline 90 & $\begin{array}{l}0,920 \\
0,638\end{array}$ & $\begin{array}{l}1,115 \\
0,814\end{array}$ & $\begin{array}{l}1,318 \\
1,006\end{array}$ & $\begin{array}{l}1,616 \\
1,302\end{array}$ & $\begin{array}{l}2,387 \\
2,135\end{array}$ & $\begin{array}{l}3,525 \\
3,500\end{array}$ & $\begin{array}{l}4,323 \\
4,570\end{array}$ & $\begin{array}{l}5,109 \\
5,416\end{array}$ & $\begin{array}{l}6,194 \\
6,839\end{array}$ \\
\hline
\end{tabular}

\section{4 - MODÈLES QDF DE RÉFÉRENCE EN RÉGIME HYDROLOGIQUE D'ÉTIAGE}

Un formalisme mathématique des abaques en VCNd et en QCNd des bassins de référence (la Jouanne à FORCE $\left(410 \mathrm{~km}^{2}\right)$, la Dunière à VAUBARLET $\left(228 \mathrm{~km}^{2}\right)$, la Couze d'Issoire à St-FLORET $\left(216 \mathrm{~km}^{2}\right)$, la Petite Sauldre à MENETREOL $\left(318 \mathrm{~km}^{2}\right)$ ) permet de faciliter leur usage. 


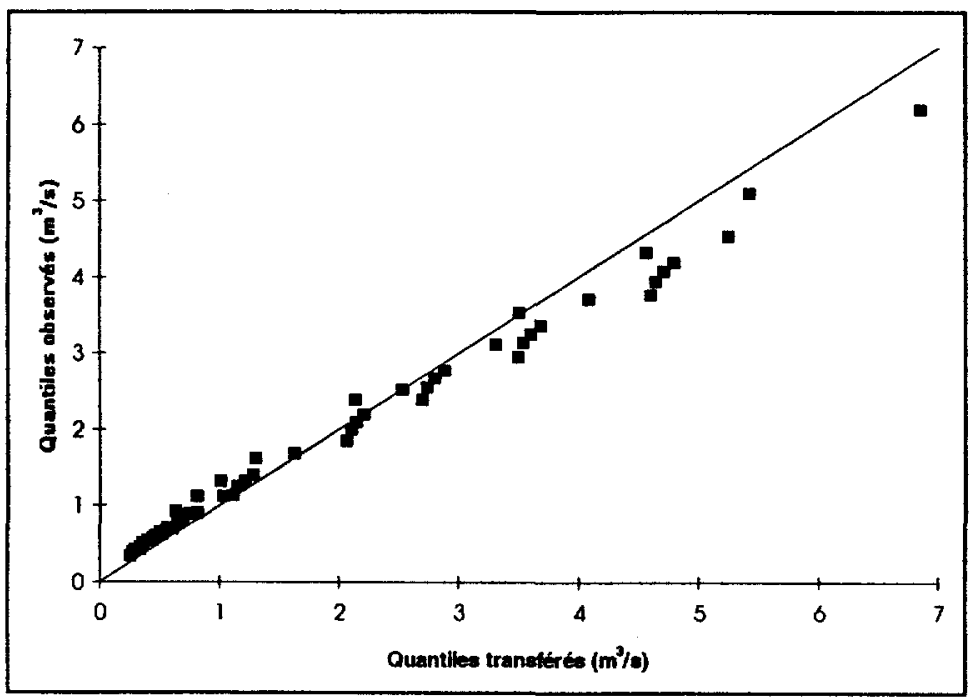

Figure 8 du tableau 3.

Figure 8, table 3.

\subsection{Modèles QdF de référence en VCNd}

Nous donnons ci-après les équations ( 1 et 2) et les paramètres (fixés une fois pour toute) relatifs aux modèles de synthèse en VCNd (tableaux 4 et 5 ).

\subsubsection{Paramètres en années sèches}

Pour chaque modèle de référence et pour chacune des plages d/De balayées, nous avons estimé les paramètres $x, y$ et $z$ des fonctions homographiques suivantes qui permettent de bien décrire la variation des coefficients a et $b$ (ex. figure 6 et figure 7) en fonction de la période moyenne de retour (T).

Tableau 4 Paramètres des modèles (en VCNd) de référence en années sèches.

Table 4 Set of reference QdF model parameters (in VCNd), for dry years.

\begin{tabular}{|c|c|c|c|c|c|c|c|}
\hline \multirow{3}{*}{\multicolumn{2}{|c|}{$\begin{array}{l}\text { Modèles de synthèse QdF d'étiage } \\
\text { (en VCNd) }\end{array}$}} & \multicolumn{6}{|c|}{ Années sèches } \\
\hline & & \multicolumn{3}{|c|}{$a_{\text {sec }}$} & \multicolumn{3}{|c|}{$b_{\text {sec }}$} \\
\hline & & \multirow{2}{*}{$\begin{array}{c}x_{1} \\
0,470 \\
1,898\end{array}$} & \multirow{2}{*}{$\begin{array}{c}\mathbf{y}_{1} \\
0,376 \\
1,341\end{array}$} & \multirow{2}{*}{$\begin{array}{c}z_{1} \\
0,236 \\
0,063\end{array}$} & \multirow{2}{*}{$\begin{array}{c}x_{2} \\
0,621 \\
0,431\end{array}$} & \multirow{2}{*}{$\begin{array}{c}\mathbf{y}_{2} \\
0,000 \\
0,074\end{array}$} & \multirow{2}{*}{$\begin{array}{c}z_{2} \\
0,125 \\
0,210\end{array}$} \\
\hline Force & $\begin{array}{c}\mathrm{d} / \mathrm{De} \leq 0,41 \\
0,41<\mathrm{d} / \mathrm{De} \leq 10\end{array}$ & & & & & & \\
\hline Vaubarlet & $\begin{array}{c}d / \mathrm{De} \leq 1 \\
1<\mathrm{d} / \mathrm{De} \leq 9\end{array}$ & $\begin{array}{l}0,761 \\
2,304\end{array}$ & $\begin{array}{l}2,255 \\
0,847\end{array}$ & $\begin{array}{l}0,200 \\
0,040\end{array}$ & $\begin{array}{l}0,538 \\
0,398\end{array}$ & $\begin{array}{l}0,229 \\
0,442\end{array}$ & $\begin{array}{l}0,195 \\
0,391\end{array}$ \\
\hline Saint-Floret & $\begin{array}{c}\mathrm{d} / \mathrm{De} \leq 0,5 \\
0,5<\mathrm{d} / \mathrm{De} \leq 6\end{array}$ & $\begin{array}{l}1,545 \\
4,037\end{array}$ & $\begin{array}{l}2,633 \\
2,698\end{array}$ & $\begin{array}{l}0,147 \\
0,055\end{array}$ & $\begin{array}{l}0,494 \\
0,452\end{array}$ & $\begin{array}{l}0,679 \\
0,643\end{array}$ & $\begin{array}{l}0,388 \\
0,437\end{array}$ \\
\hline Menetreol & $\begin{array}{c}\mathrm{d} / \mathrm{De} \leq 0,28 \\
0,28<\mathrm{d} / \mathrm{De} \leq 4,5\end{array}$ & $\begin{array}{l}6,726 \\
3,936\end{array}$ & $\begin{array}{l}4,259 \\
2,632\end{array}$ & $\begin{array}{l}0,137 \\
0,017\end{array}$ & $\begin{array}{l}0,524 \\
0,513\end{array}$ & $\begin{array}{l}0,981 \\
1,016\end{array}$ & $\begin{array}{l}0,499 \\
0,532\end{array}$ \\
\hline
\end{tabular}




$$
a_{\text {sec }}=\frac{1}{x_{1} \cdot T+y_{1}}+z_{1} \quad b_{\text {sec }}=\frac{1}{x_{2} \cdot T+y_{2}}+z_{2}
$$

Les quantiles secs VCN(T, d) d'un bassin versant peuvent être estimés par la relation (1) :

$\operatorname{VCN}(T, d)=\left\{\left(\frac{1}{x_{1} \cdot T+y_{1}}+z_{1}\right) \cdot \frac{d}{D e}+\left(\frac{1}{x_{2} \cdot T+y_{2}}+z_{2}\right)\right\} \cdot \operatorname{VCN}(2,1)_{\begin{array}{c}\text { bassin } \\ \text { versant } \\ \text { étudié }\end{array}}$

où De et $\mathrm{d}$ sont exprimés dans la même unité.

\subsubsection{Paramètres en années humides}

De même que précédemment, sont estimés les paramètres $x, y$ et $z$ pour chacun des coefficients $a$ et $b$, en prenant le formalisme mathématique ci-après, mieux adapté à la variation de $a(T)$ et $b(T)$.

$$
a_{\text {numide }}=x_{3} \cdot \sqrt{T}+\frac{y_{3}}{T}+z_{3} \quad b_{\text {humide }}=x_{4} \cdot \sqrt{T}+\frac{y_{4}}{T}+z_{4}
$$

Tableau 5 Paramètres des modèles (en VCNd) de référence en années humides.

Table 5 Set of reference QdF model parameters (in VCNd), for humid years.

\begin{tabular}{|l|c|c|c|c|c|c|c|}
\hline \multirow{2}{*}{$\begin{array}{l}\text { Modèles de synthèse QdF d'étiage } \\
\text { (en VCNd) }\end{array}$} & \multicolumn{6}{|c|}{ Années humides } \\
\cline { 3 - 8 } & & \multicolumn{3}{|c|}{$a_{\text {humide }}$} & \multicolumn{3}{c|}{$b_{\text {humide }}$} \\
\cline { 3 - 8 } & $\mathbf{x}_{\mathbf{3}}$ & $\mathbf{y}_{\mathbf{3}}$ & $\mathbf{z}_{\mathbf{3}}$ & $\mathbf{x}_{\mathbf{4}}$ & $\mathbf{y}_{\mathbf{4}}$ & $\mathbf{z}_{\mathbf{4}}$ \\
\hline Force & $\mathrm{d} / \mathrm{De} \leq 0,41$ & 0,144 & $-3,028$ & 1,894 & 0,664 & $-3,684$ & 1,218 \\
& $0,41<\mathrm{d} / \mathrm{De} \leq 12$ & 0,066 & $-0,748$ & 0,432 & 0,700 & $-4,733$ & 1,898 \\
Vaubarlet & $\mathrm{d} / \mathrm{De} \leq 8,72$ & 0,069 & $-0,698$ & 0,405 & 0,300 & $-2,990$ & 1,728 \\
Saint-Floret & $\mathrm{d} / \mathrm{De} \leq 1$ & 0,029 & $-0,432$ & 0,379 & 0,130 & $-1,816$ & 1,498 \\
& $1<\mathrm{d} / \mathrm{De} \leq 6$ & 0,032 & $-0,135$ & 0,161 & 0,140 & $-1,970$ & 1,644 \\
Menetreol & $\mathrm{d} / \mathrm{De} \leq 4$ & 0,026 & $-0,329$ & 0,201 & 0,072 & $-1,228$ & 1,384 \\
\hline
\end{tabular}

La relation (2) permet d'estimer les quantiles humides VCN(T, d) d'un bassin versant.

$\operatorname{VCN}(T, d)=\left\{\left(x_{3} \cdot \sqrt{T}+\frac{y_{3}}{T}+z_{3}\right) \cdot \frac{d}{D e}+\left(x_{4} \cdot \sqrt{T}+\frac{y_{4}}{T}+z_{4}\right)\right\} \cdot \operatorname{VCN}(2,1)_{\substack{\text { bassin } \\ \text { versant } \\ \text { étudié }}}$

\subsection{Modèles QdF de référence en QCNd}

Nous donnons ci-après les équations (3) et (4) relatives aux modèles en QCNd ainsi que les paramètres correspondants (tableaux 6 et 7).

\subsubsection{Paramètres en années sèches}

$$
a_{\text {sec }}=\frac{1}{x_{1} \cdot T+y_{1}}+z_{1} \quad b_{\text {sec }}=\frac{1}{x_{2} \cdot T+y_{2}}+z_{2}
$$


Tableau 6 Paramètres des modèles (en QCNd) de référence en années sèches. Table 6 Set of reference QdF model parameters (in QCNd), for dry years.

\begin{tabular}{|c|c|c|c|c|c|c|c|}
\hline \multirow{3}{*}{\multicolumn{2}{|c|}{$\begin{array}{c}\text { Modèles de synthèse QdF d'étiage } \\
\text { (en QCNd) }\end{array}$}} & \multicolumn{6}{|c|}{ Années sèches } \\
\hline & & \multicolumn{3}{|c|}{$a_{\text {sec }}$} & \multicolumn{3}{|c|}{$b_{\text {sec }}$} \\
\hline & & $x_{1}$ & $y_{1}$ & $z_{1}$ & $x_{2}$ & $y_{2}$ & $z_{2}$ \\
\hline Force & $\begin{array}{c}\mathrm{d} / \mathrm{De} \leq 0,41 \\
0,41<\mathrm{d} / \mathrm{De} \leq 3,42\end{array}$ & $\begin{array}{l}0,250 \\
0,571\end{array}$ & $\begin{array}{l}0,237 \\
0,209\end{array}$ & $\begin{array}{l}0,301 \\
0,175\end{array}$ & $\begin{array}{l}0,725 \\
0,539\end{array}$ & $\begin{array}{r}-0,123 \\
0,004\end{array}$ & $\begin{array}{l}0,141 \\
0,231\end{array}$ \\
\hline Vaubarlet & $\mathrm{d} / \mathrm{De} \leq 4,1$ & 0,637 & 0,131 & 0,203 & 0,655 & 0,337 & 0,287 \\
\hline Saint-Floret & $\mathrm{d} / \mathrm{De} \leq 2,9$ & 0,757 & 0,574 & 0,154 & 0,519 & 0,786 & 0,419 \\
\hline Menetreol & $\begin{array}{c}\mathrm{d} / \mathrm{De} \leq 0,21 \\
0,21<\mathrm{d} / \mathrm{De} \leq 2,1\end{array}$ & $\begin{array}{l}3,114 \\
1,141\end{array}$ & $\begin{array}{l}2,193 \\
0,323\end{array}$ & $\begin{array}{l}0,251 \\
0,037\end{array}$ & $\begin{array}{l}0,692 \\
0,438\end{array}$ & $\begin{array}{l}0,780 \\
1,499\end{array}$ & $\begin{array}{l}0,527 \\
0,537\end{array}$ \\
\hline
\end{tabular}

Les quantiles secs QCN(T, d) d'un bassin versant donné peuvent être estimés par la relation (3) :

$\operatorname{QCN}(T, d)=\left\{\left(\frac{1}{x_{1} \cdot T+y_{1}}+z_{1}\right) \cdot \frac{d}{D e}+\left(\frac{1}{x_{2} \cdot T+y_{2}}+z_{2}\right)\right\} \cdot \operatorname{QCN}(2,1)_{\substack{\text { bassin } \\ \text { versant } \\ \text { étudié }}}$

\subsubsection{Paramètres en années humides}

$$
a_{\text {humide }}=x_{3} \cdot T^{1,5}+\frac{y_{3}}{T^{\theta}}+z_{3} \quad b_{\text {humide }}=x_{4} \cdot T^{1,5}+\frac{y_{4}}{T^{\theta}}+z_{4}
$$

Tableau 7 Paramètres des modèles (en QCNd) de référence en années sèches.

Table 7 Set of reference QdF model parameters (in QCNd), for dry years.

\begin{tabular}{|c|c|c|c|c|c|c|c|c|}
\hline \multirow{3}{*}{\multicolumn{2}{|c|}{$\begin{array}{c}\text { Modèles de synthèse QdF } \\
\text { d'étiage } \\
\text { (en aCNd) }\end{array}$}} & \multicolumn{7}{|c|}{ Années humides } \\
\hline & & \multicolumn{3}{|c|}{$a_{\text {humide }}$} & \multicolumn{3}{|c|}{$b_{\text {humide }}$} & \multirow{2}{*}{$\theta$} \\
\hline & & \multirow{2}{*}{$\begin{array}{c}x_{3} \\
0,0044 \\
0,0040\end{array}$} & \multirow{2}{*}{ 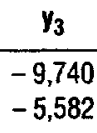 } & \multirow{2}{*}{$\begin{array}{c}z_{3} \\
4,095 \\
2,855\end{array}$} & \multirow{2}{*}{$\begin{array}{l}x_{4} \\
0,0052 \\
0,0051\end{array}$} & \multirow{2}{*}{\begin{tabular}{|c|}
$\mathbf{y}_{\mathbf{4}}$ \\
$-10,462$ \\
$-10,53 \uparrow$
\end{tabular}} & \multirow{2}{*}{$\begin{array}{l}z_{\mathbf{4}} \\
4,073 \\
4,150\end{array}$} & \\
\hline Force & $\begin{array}{c}\mathrm{d} / \mathrm{De} \leq 0,82 \\
0,82<\mathrm{d} / \mathrm{De} \leq 4,1\end{array}$ & & & & & & & $\begin{array}{l}1 \\
1\end{array}$ \\
\hline Vaubarlet & $\begin{array}{c}\mathrm{d} / \mathrm{De} \leq 1,34 \\
2,34<\mathrm{d} / \mathrm{De} \leq 5,4\end{array}$ & $\begin{array}{l}0,0007 \\
0,0053\end{array}$ & $\begin{array}{l}-2,116 \\
-6,397\end{array}$ & $\begin{array}{l}1,969 \\
4,535\end{array}$ & $\begin{array}{r}0,0023 \\
-0,0030\end{array}$ & $\begin{array}{r}-4,860 \\
3,505\end{array}$ & $\begin{array}{r}3,538 \\
-1,087\end{array}$ & $\begin{array}{l}0,6 \\
0,6\end{array}$ \\
\hline Saint-Floret & $\begin{array}{c}\mathrm{d} / \mathrm{De} \leq 0,5 \\
0,5<\mathrm{d} / \mathrm{De} \leq 2,9\end{array}$ & $\begin{array}{l}0,0007 \\
0,0015\end{array}$ & $\begin{array}{l}-1,405 \\
-2,385\end{array}$ & $\begin{array}{l}1,387 \\
1,995\end{array}$ & $\begin{array}{l}0,0010 \\
0,0005\end{array}$ & $\begin{array}{l}-2,044 \\
-1,769\end{array}$ & $\begin{array}{l}2,161 \\
1,945\end{array}$ & $\begin{array}{l}0,6 \\
0,6\end{array}$ \\
\hline Menetreol & $\begin{array}{c}\mathrm{d} / D \mathrm{De} \leq 1,05 \\
1,05<\mathrm{d} / \mathrm{De} \leq 2,5\end{array}$ & $\begin{array}{l}0,0004 \\
0,0022\end{array}$ & $\begin{array}{l}-0,793 \\
-4,530\end{array}$ & $\begin{array}{l}0,8147 \\
2,869\end{array}$ & $\begin{array}{r}0,0004 \\
-0,0021\end{array}$ & $\begin{array}{r}-1,448 \\
1,963\end{array}$ & $\begin{array}{r}1,812 \\
-0,1341\end{array}$ & $\begin{array}{l}0,6 \\
0,6\end{array}$ \\
\hline
\end{tabular}

Les quantiles humides $\mathrm{QCN}(\mathrm{T}, \mathrm{d})$ d'un bassin versant donné se déduisent de la relation (4):

$$
\operatorname{QCN}(T, d)=\left\{\left(x_{3} \cdot T^{1,5}+\frac{y_{3}}{T^{\theta}}+z_{3}\right) \cdot \frac{d}{D e}+\left(x_{4} \cdot T^{1,5}+\frac{y_{4}}{T^{\theta}}+z_{4}\right)\right\} \cdot \operatorname{QCN}(2,1)_{\begin{array}{c}
\text { bassin } \\
\text { versant } \\
\text { étudié }
\end{array}}
$$


avec $\mathrm{QCN}(2,1)={ }^{1} \mathrm{VCN}(2,1)$

Nous rappelons que $\operatorname{VCN}(2,1)$ et De sont les seuls paramètres d'entrée nécessaires aux quatre modèles synthétiques de référence en VCNd et QCNd.

\section{5 - PRÉSENTATION DE LA TYPOLOGIE DES ÉTIAGES DU BASSIN DE LA LOIRE}

\subsection{Cohérence des modélisations en VCNd et QCNd}

Nous présentons, comme exemple, les résultats des modélisations du bassin versant de l'Allier à Monistrol d'Allier $\left(S=988 \mathrm{~km}^{2}\right)$. L'étude analytique de la chronique de débit journalier (1947-1988), peu influencée par la mise en service (1986) du barrage de Naussac (soutien d'étiage), permet d'identifier le modèle $\mathrm{QdF}$ de référence de FORCE (on notera leur éloignement géographique, figure 11 et figure 4) ainsi que les descripteurs locaux de régime $\mathrm{De}=15,2 \mathrm{j}$ et $\operatorname{VCN}(2,1)=0,845 \mathrm{~m}^{3} / \mathrm{s}$. Les tableaux 8 et 9 ainsi que les figures 9 et 10 correspondantes permettent de comparer respectivement les résultats relatifs aux quantiles de VCNd et QCNd, observés et modélisés.

Tableau 8 Bassin de l'Allier à Monistrol d'Allier - VCN $(T, d)$ observés et modélisés.

Table $8 \quad$ Allier's basin at Monistrol d'Allier - observed and modelling VCN(T, d).

\begin{tabular}{|c|c|c|c|c|c|c|c|c|c|}
\hline \multirow{6}{*}{$\begin{array}{c}\text { Durée } \\
\text { continue } \\
d(j)\end{array}$} & \multicolumn{9}{|c|}{$\operatorname{VCN}(T, d)\left(m^{3} / s\right)$} \\
\hline & \multicolumn{5}{|c|}{ Quantiles secs } & \multicolumn{4}{|c|}{ Quantiles humides } \\
\hline & \multicolumn{9}{|c|}{$T(a n)$} \\
\hline & 50 & 20 & 10 & 5 & 2 & 5 & 10 & 20 & 50 \\
\hline & \multicolumn{9}{|c|}{$\mathrm{U}_{\mathrm{F}}$} \\
\hline & $-2,055$ & $-1,64$ & $-1,28$ & $-0,84$ & 0 & 0,84 & 1,28 & 1,64 & 2,055 \\
\hline 1 & $\begin{array}{l}0,138 \\
0,148\end{array}$ & $\begin{array}{l}0,199 \\
0,192\end{array}$ & $\begin{array}{l}0,273 \\
0,266\end{array}$ & $\begin{array}{l}0,403 \\
0,411\end{array}$ & $\begin{array}{l}0,845 \\
0,841\end{array}$ & $\begin{array}{l}1,772 \\
1,751\end{array}$ & $\begin{array}{l}2,612 \\
2,606\end{array}$ & $\begin{array}{l}3,588 \\
3,515\end{array}$ & $\begin{array}{l}5,173 \\
5,093\end{array}$ \\
\hline 3 & $\begin{array}{l}0,161 \\
0,179\end{array}$ & $\begin{array}{l}0,231 \\
0,230\end{array}$ & $\begin{array}{l}0,317 \\
0,314\end{array}$ & $\begin{array}{l}0,465 \\
0,478\end{array}$ & $\begin{array}{l}0,967 \\
0,952\end{array}$ & $\begin{array}{l}2,011 \\
1,930\end{array}$ & $\begin{array}{l}2,951 \\
2,834\end{array}$ & $\begin{array}{l}4,039 \\
3,781\end{array}$ & $\begin{array}{l}5,801 \\
5,410\end{array}$ \\
\hline 6 & $\begin{array}{l}0,192 \\
0,226\end{array}$ & $\begin{array}{l}0,273 \\
0,286\end{array}$ & $\begin{array}{l}0,369 \\
0,386\end{array}$ & $\begin{array}{l}0,533 \\
0,579\end{array}$ & $\begin{array}{l}1,079 \\
1,118\end{array}$ & $\begin{array}{l}2,183 \\
2,198\end{array}$ & $\begin{array}{l}3,158 \\
3,175\end{array}$ & $\begin{array}{l}4,271 \\
4,179\end{array}$ & $\begin{array}{l}6,051 \\
5,886\end{array}$ \\
\hline 10 & $\begin{array}{l}0,229 \\
0,257\end{array}$ & $\begin{array}{l}0,320 \\
0,324\end{array}$ & $\begin{array}{l}0,427 \\
0,433\end{array}$ & $\begin{array}{l}0,609 \\
0,643\end{array}$ & $\begin{array}{l}1,196 \\
1,223\end{array}$ & $\begin{array}{l}2,350 \\
2,366\end{array}$ & $\begin{array}{l}3,347 \\
3,389\end{array}$ & $\begin{array}{l}4,471 \\
4,433\end{array}$ & $\begin{array}{l}6,241 \\
6,198\end{array}$ \\
\hline 30 & $\begin{array}{l}0,336 \\
0,339\end{array}$ & $\begin{array}{l}0,460 \\
0,422\end{array}$ & $\begin{array}{l}0,606 \\
0,557\end{array}$ & $\begin{array}{l}0,847 \\
0,816\end{array}$ & $\begin{array}{l}1,606 \\
1,510\end{array}$ & $\begin{array}{l}3,047 \\
2,844\end{array}$ & $\begin{array}{l}4,260 \\
4,018\end{array}$ & $\begin{array}{l}5,605 \\
5,199\end{array}$ & $\begin{array}{l}7,690 \\
7,180\end{array}$ \\
\hline 90 & $\begin{array}{l}0,630 \\
0,584\end{array}$ & $\begin{array}{l}0,840 \\
0,717\end{array}$ & $\begin{array}{l}1,079 \\
0,932\end{array}$ & $\begin{array}{l}1,466 \\
1,334\end{array}$ & $\begin{array}{l}2,627 \\
2,369\end{array}$ & $\begin{array}{l}4,711 \\
4,278\end{array}$ & $\begin{array}{l}6,396 \\
5,906\end{array}$ & $\begin{array}{l}8,214 \\
7,500\end{array}$ & $\begin{array}{l}10,960 \\
10,128\end{array}$ \\
\hline
\end{tabular}

1 À partir des chroniques de débit journalier les échantillons de VCNd(1 j) et QCNd(1 j) ne sont pas différenciés, ce qui est aussi conforme avec la réalité des régimes de basses eaux observés. 
Les résultats obtenus par modélisation sont cohérents avec ceux déduits des observations. Le concept de modèle de référence d'une famille hydrologique de bassins versants peut ainsi de site en site être validé.

Tableau 9 Bassin de l'Allier à Monistrol d'Allier - QCN(T, d) observés et modélisés. Table 9 Allier's basin at Monistrol d'Allier - observed and modelling QCN $(T, d)$.

\begin{tabular}{|c|c|c|c|c|c|c|c|c|c|}
\hline \multirow{6}{*}{$\begin{array}{c}\text { Durée } \\
\text { continue } \\
\mathrm{d}(\mathrm{i})\end{array}$} & \multicolumn{9}{|c|}{$\operatorname{aCN}(T, d)\left(m^{3} / s\right)$} \\
\hline & \multicolumn{5}{|c|}{ Quantiles secs } & \multicolumn{4}{|c|}{ Quantiles humides } \\
\hline & \multicolumn{9}{|c|}{$T$ (an) } \\
\hline & 50 & 20 & 10 & 5 & 2 & 5 & 10 & 20 & 50 \\
\hline & \multicolumn{9}{|c|}{$U_{F}$} \\
\hline & $-2,055$ & $-1,64$ & $-1,28$ & $-0,84$ & 0 & 0,84 & 1,28 & 1,64 & 2,055 \\
\hline 1 & $\begin{array}{l}0,138 \\
0,164\end{array}$ & $\begin{array}{l}0,199 \\
0,205\end{array}$ & $\begin{array}{l}0,273 \\
0,275\end{array}$ & $\begin{array}{l}0,403 \\
0,415\end{array}$ & $\begin{array}{l}0,845 \\
0,848\end{array}$ & $\begin{array}{l}1,772 \\
1,845\end{array}$ & $\begin{array}{l}2,612 \\
2,878\end{array}$ & $\begin{array}{l}3,588 \\
3,617\end{array}$ & $\begin{array}{l}5,173 \\
5,130\end{array}$ \\
\hline 3 & $\begin{array}{l}0,177 \\
0,206\end{array}$ & $\begin{array}{l}0,254 \\
0,260\end{array}$ & $\begin{array}{l}0,347 \\
0,349\end{array}$ & $\begin{array}{l}0,509 \\
0,523\end{array}$ & $\begin{array}{l}1,055 \\
1,032\end{array}$ & $\begin{array}{l}2,188 \\
2,089\end{array}$ & $\begin{array}{l}3,206 \\
3,241\end{array}$ & $\begin{array}{l}4,382 \\
4,062\end{array}$ & $\begin{array}{l}6,282 \\
5,735\end{array}$ \\
\hline 6 & $\begin{array}{l}0,249 \\
0,269\end{array}$ & $\begin{array}{l}0,346 \\
0,342\end{array}$ & $\begin{array}{l}0,460 \\
0,460\end{array}$ & $\begin{array}{l}0,652 \\
0,685\end{array}$ & $\begin{array}{l}1,269 \\
1,309\end{array}$ & $\begin{array}{l}2,468 \\
2,455\end{array}$ & $\begin{array}{l}3,496 \\
3,784\end{array}$ & $\begin{array}{l}4,650 \\
4,729\end{array}$ & $\begin{array}{l}6,460 \\
6,643\end{array}$ \\
\hline 10 & $\begin{array}{l}0,315 \\
0,343\end{array}$ & $\begin{array}{l}0,430 \\
0,418\end{array}$ & $\begin{array}{l}0,564 \\
0,543\end{array}$ & $\begin{array}{l}0,785 \\
0,787\end{array}$ & $\begin{array}{l}1,476 \\
1,485\end{array}$ & $\begin{array}{l}2,775 \\
2,944\end{array}$ & $\begin{array}{l}3,863 \\
4,509\end{array}$ & $\begin{array}{l}5,065 \\
5,618\end{array}$ & $\begin{array}{l}6,920 \\
7,854\end{array}$ \\
\hline 30 & $\begin{array}{l}0,578 \\
0,576\end{array}$ & $\begin{array}{l}0,788 \\
0,709\end{array}$ & $\begin{array}{l}1,031 \\
0,926\end{array}$ & $\begin{array}{l}1,431 \\
1,345\end{array}$ & $\begin{array}{l}2,678 \\
2,503\end{array}$ & $\begin{array}{l}5,011 \\
4,750\end{array}$ & $\begin{array}{l}6,958 \\
6,796\end{array}$ & $\begin{array}{l}9,102 \\
8,342\end{array}$ & $\begin{array}{l}12,404 \\
11,790\end{array}$ \\
\hline
\end{tabular}

\subsection{Typologie des étiages du bassin de la Loire}

Les quatre modèles QdF d'étiage, appliqués aux différents sites sélectionnés, permettent de donner une représentation (figure 11) de la typologie des régimes d'étiage observés dans le bassin hydrographique de la Loire. Par ailleurs, ces résultats ne permettent pas de privilégier, pour chacune des onze unités géographiques homogènes de gestion (figure 12) que représentent les hydroécorégions (Cemagref, CNRS, 1993), l'usage a priori de l'un des quatre modèles QdF de référence. Autrement dit, la nature géologique des bassins versants (par exemple) ne semble pas pour l'instant permettre un choix direct du modèle QdF d'étiage de référence. II conviendra ultérieurement, en y associant d'autres études de régionalisation, d'expliciter le critère de choix (cf. § 3.1) à partir des caractéristiques physiographiques des bassins versants prises à l'intérieur d'une même typologie des régimes d'étiage.

\section{6 - APPLICATION DES MODĖLES QDF AUX ÉTIAGES DU CRISU-ALB (ROUMANIE)}

\subsection{Résultats généraux}

Le Crisu-Alb prend sa source à environ $1800 \mathrm{~m}$ d'altitude en Transylvanie, il coule vers l'ouest de la Roumanie et traverse une région aux hauteurs boisées et 


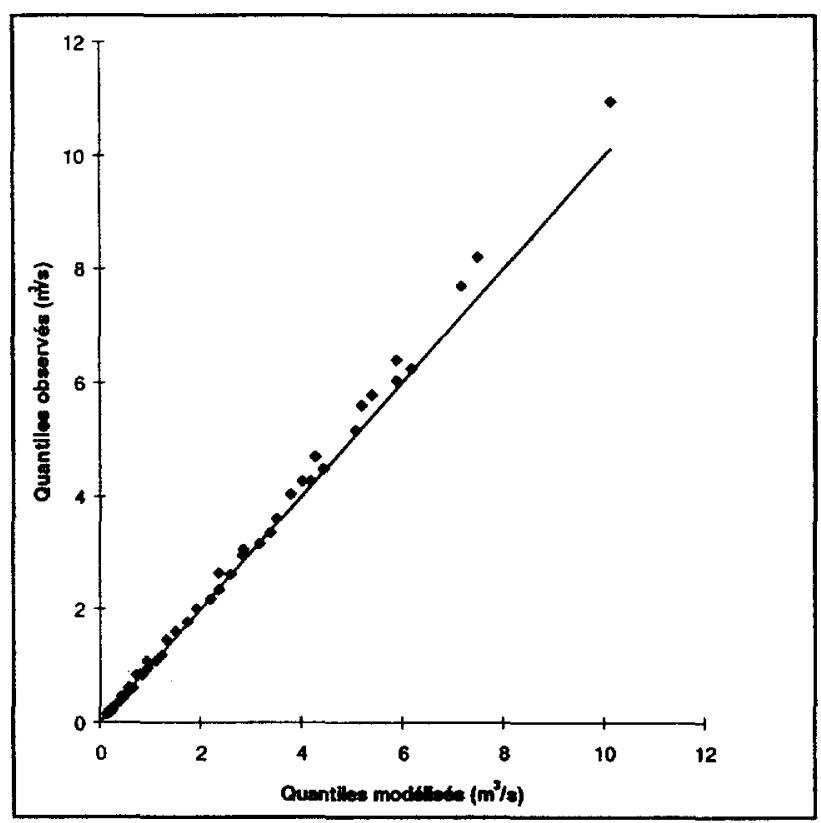

Figure 9 du tableau 8 " VCN(T, d) ».

Figure 9, table 8 "VCN(T,d)".

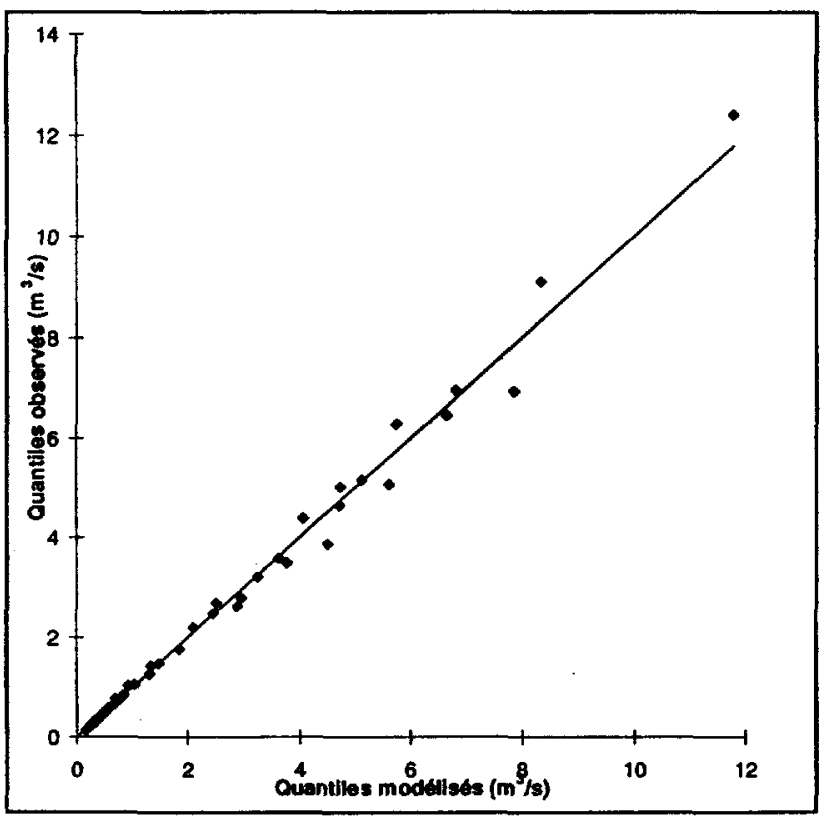

Figure 10 du tableau 9 "QCN(T,d) ".

Figure 10, table 9 "QCN(T,d)". 


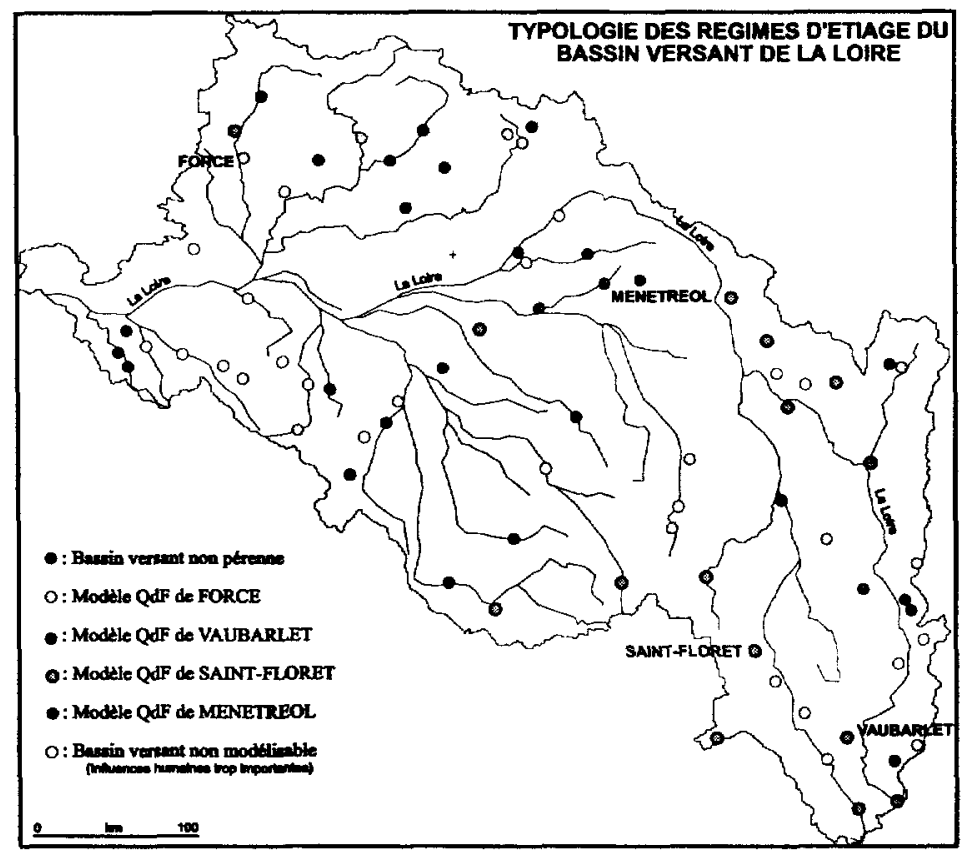

Figure 11 Typologie des étiages observés du bassin de la Loire.

Observed low flows typology of Loire's basin.

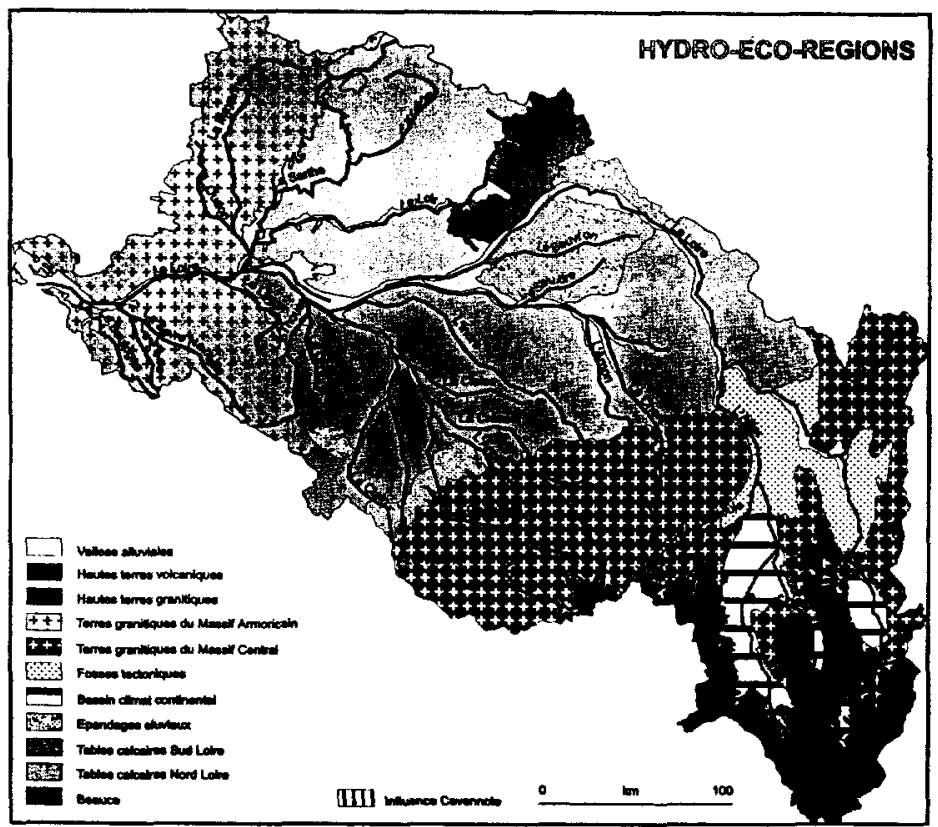

Figure 12 Hydroécorégions du bassin de la Loire.

Ecohydrological region of Loire's basin. 
aux vallées à vocation maraîchère. Les quatre modèles de référence établis sur le bassin hydrographique de la Loire ont été appliqués sur 12 des sites d'observation du bassin hydrographique du Crisu Alb $\left(S=3000 \mathrm{~km}^{2}\right)$ en Roumanie (figure 13).

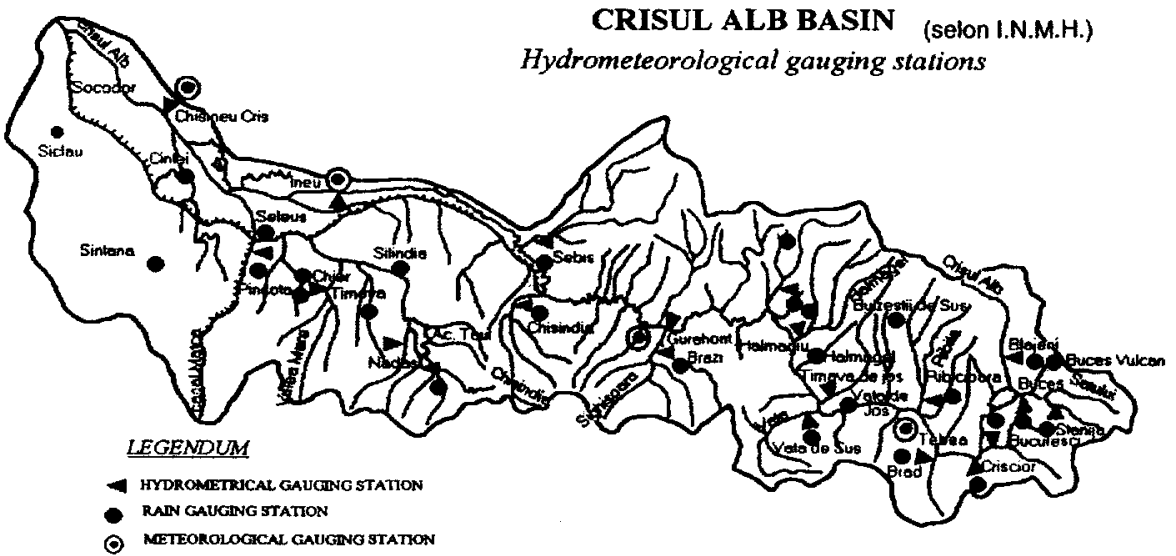

Figure 13 Bassins observés du Crisu-Alb.

Observed basins in Crisu-Alb.

Les régimes hydrologiques naturels des 12 bassins versants traités (tableau 10) ont pu être répartis, selon le critère lié à l'écart-type de la loi ajustée sur les $V C N d=1 \mathrm{j}$ (cf. $\$ 3.1$ ), parmi les 4 familles hydrologiques identifiées sur le bassin de la Loire.

Tableau 10 Sites observés retenus.

Table to Observed and selected basins.

\begin{tabular}{|c|c|c|c|c|c|c|c|}
\hline \multicolumn{8}{|c|}{ Bassin hydrographique du CRISU-ALB (S = $3000 \mathrm{~km}^{2}$ ) } \\
\hline Rivière & $\begin{array}{c}\text { Station } \\
\text { hydromélrique } \\
\text { (site observé) }\end{array}$ & Code & $\begin{array}{c}\text { Surface } \\
\left(\mathrm{km}^{2}\right)\end{array}$ & $\begin{array}{c}\text { Chronique } \\
\text { débit } \\
\text { journalier }\end{array}$ & $\begin{array}{c}\text { Ėcart-type (s) } \\
\text { loi LN sur } \\
\text { VCNd = 1 j }\end{array}$ & $\begin{array}{l}\text { Modèle } \\
\text { Odf de } \\
\text { référence }\end{array}$ & $\begin{array}{c}\text { Critère de } \\
\text { Nash (\%) tous } \\
\text { quantiles } \\
\text { confondus }\end{array}$ \\
\hline Banestilor & HALMAGIU & 30605 & 108,0 & $1967-1993$ & 0,35 & Menetreol & 98,94 \\
\hline Chisindia & CHISINDIA & 31105 & 46,6 & $1978-1993$ & 0,38 & Menetreol & 94,61 \\
\hline Crisu Alb & BLAJENI & 30105 & 106,0 & $1968-1994$ & 0,80 & Vaubarlet & 90,12 \\
\hline Crisu Alb & CRISCIOR & 30110 & 333,0 & $1961-1993$ & 0,82 & Force & 97,52 \\
\hline Crisu Alb & GURAHONT & 30115 & 1581,0 & $1953-1992$ & 0,58 & Vaubarlet & 98,55 \\
\hline Halmagel & HALMAGEL & 30705 & 67,0 & $1965-1993$ & 0,41 & St-Fioret & 99,69 \\
\hline Ribita & RIBICIOARA & 30405 & 39,2 & $1978-1993$ & 0,35 & Menetreol & 85,67 \\
\hline Sebes & SEBES & 31205 & 208,0 & $1954-1993$ & 0,48 & St-Floret & 84,26 \\
\hline Sighisoara & BRAZI & 30905 & 120,0 & $1965-1993$ & 0,66 & Vaubarlet & 98,60 \\
\hline Valea De Lazuri & VIRFURILE & 30805 & 78,6 & 1978-1993 & 0,45 & St-Foret & 95,50 \\
\hline Valea Satului & BUCES & 30205 & 104,0 & $1978-1993$ & 0,67 & Vaubarlet & 93,32 \\
\hline Vata & VATA DE SUS & 30505 & 78,6 & $1978-1993$ & 0,43 & St-Floret & 97,53 \\
\hline \multicolumn{8}{|c|}{ Moyenne des critères de Nash (tous modèles et quantiles confondus * VCN(T, d) N) } \\
\hline \multicolumn{4}{|c|}{ BASSIN HYDROGRAPHIQUE DE LA LOIRE } & \multicolumn{4}{|c|}{ BASSIN HYDROGRAPHIOUE DU CRISU-ALB } \\
\hline \multicolumn{4}{|c|}{93,06 (57 sites) } & \multicolumn{4}{|c|}{94,94 (12 sites) } \\
\hline
\end{tabular}


Pour les 12 sites étudiés du Crisu-Alb, le critère de Nash a été établi tous quantiles naturels ( $N$ valeurs) en VCNd confondus (secs et humides), pour les périodes moyennes de retour $\mathrm{T}$ ( 2 ans à 50 ans) et les durées $\mathrm{d}(1 \mathrm{j}$ à $30 \mathrm{j}$ ) étudiées.

$$
\text { Nash\% }=100 \cdot\left(1-\frac{\Sigma\left(Q(T, d)_{\text {observé }}-Q(T, d)_{\text {modélisé }}\right)^{2}}{\Sigma\left(Q(T, d)_{\text {observé }}-\frac{\Sigma(T, d)_{\text {observé }}}{N}\right)^{2}}\right)
$$

Son ordre de grandeur moyen (tableau 10) est comparable à celui obtenu (non corrigé du nombre de bassins, de d étendue à 90 jours, etc.) pour les sites sélectionnés du bassin de la Loire. Plus précisément, les coefficients de Nash (tableau 10) et les illustrations graphiques ci-après (figure 14 à figure 23) permettent de confirmer l'objectivité des modélisations (en VCNd et QCNd) réalisées sur 12 des sites observés du bassin hydrographique du Crisu-Alb.

\subsection{Résultats des modélisations en VCNd sur quelques sites}

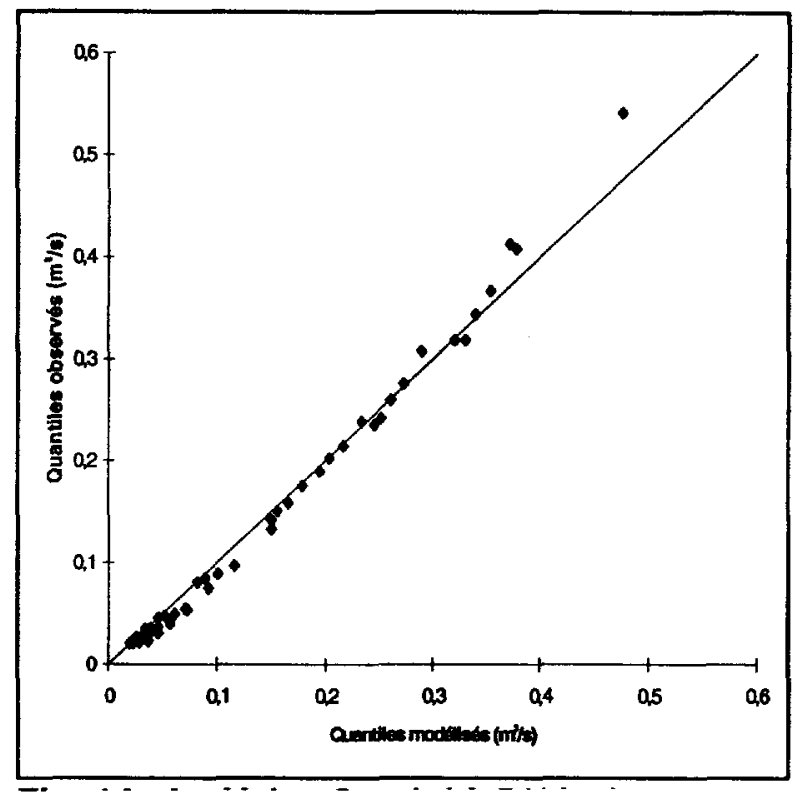

Figure 14 Le Valea Satului à BUCES « VCN(T, d) ».

Valea's basin at BUCES "VCN $(T, d)$ ".

$\left(\mathrm{De}=10,3\right.$ j et $\left.\operatorname{VCN}(2,1)=0,081 \mathrm{~m}^{3} / \mathrm{s}\right)$. 


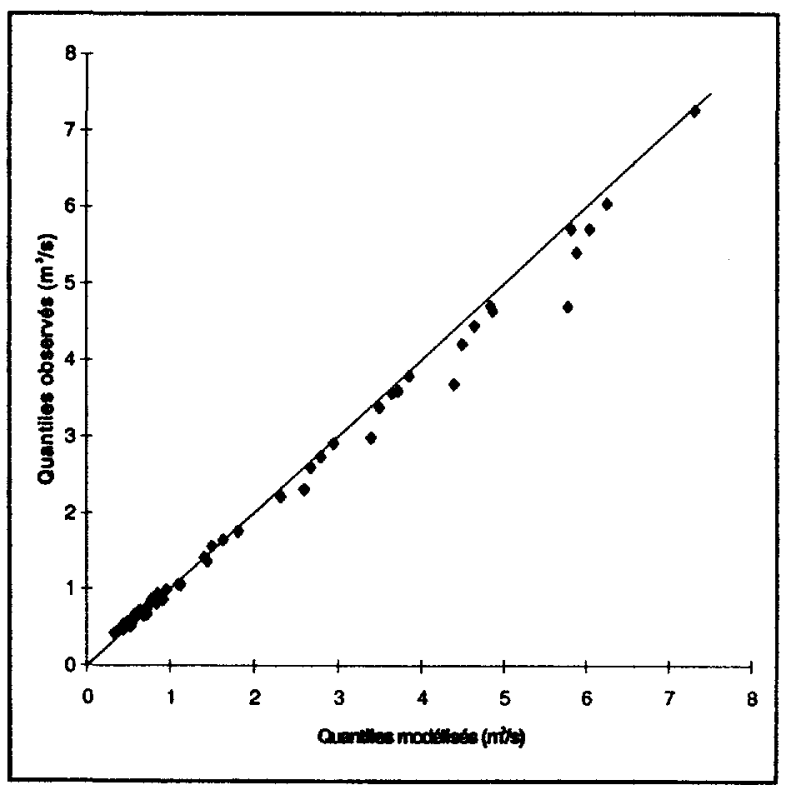

Figure 15 Crisu-Alb à GURAHONT « VCN(T, d) ».

Crisu-Alb's basin at GURAHONT "VCN $(T, d)$ ". (De $=16,4 \mathrm{j}$ et $\operatorname{VCN}(2,1)=1,419 \mathrm{~m}^{3} / \mathrm{s}$ ).

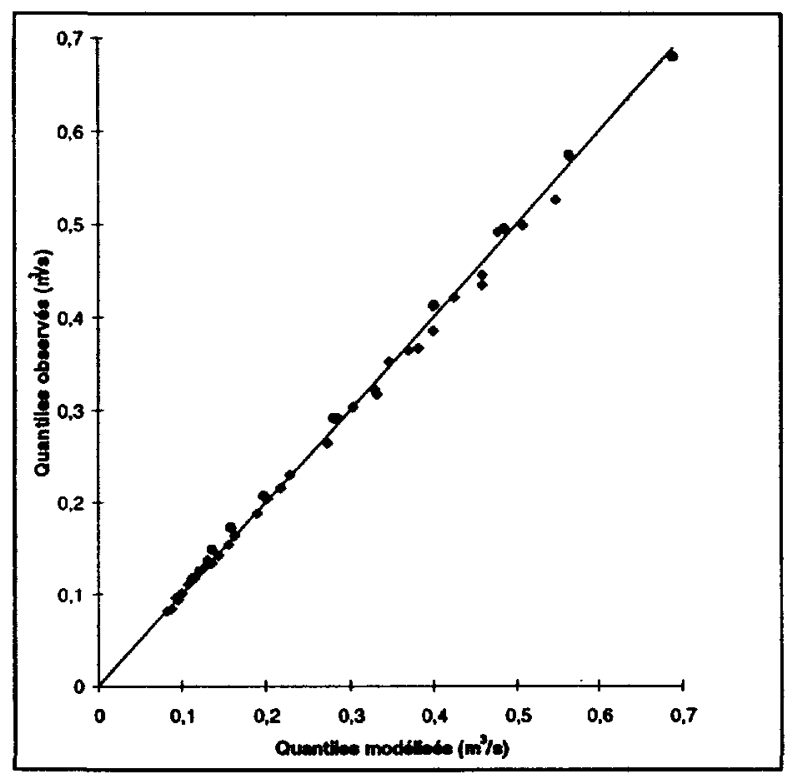

Figure 16 Halmagel à HALMAGEL « VCN $(T, d)$ ».

Halmagel's basin at HALMAGEL “VCN(T, d)".

(De $=10,8$ i et VCN $(2,1)=0,188 \mathrm{~m}^{3} / \mathrm{s}$ ). 


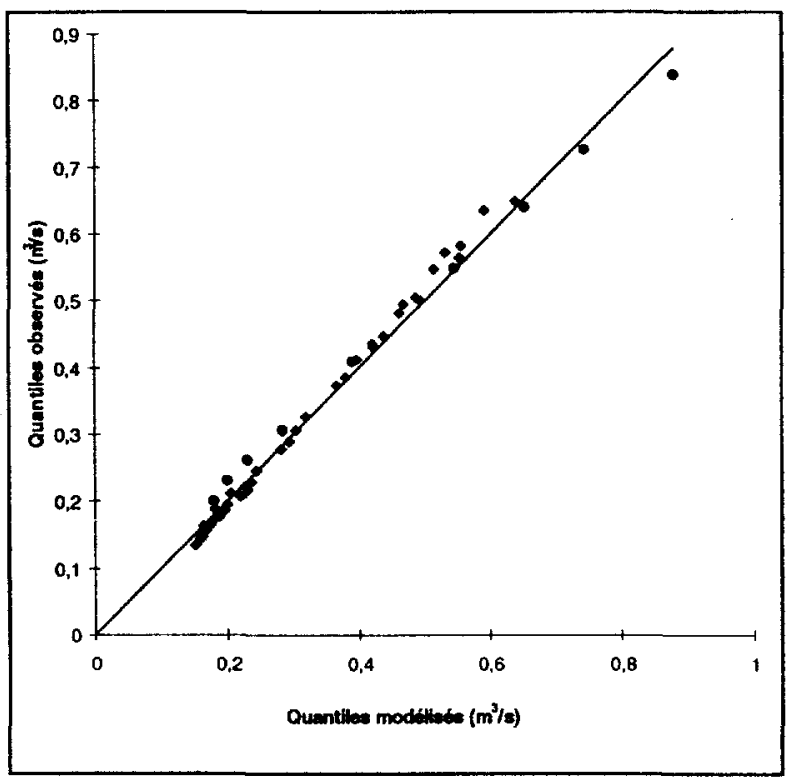

Figure 17 Banestilor à HALMAGIU « VCN(T, d) ".

Banestilor's basin at HALMAGIU "VCN(T, d)".

(De $=8,8$ i et $\operatorname{VCN}(2,1)=0,278 \mathrm{~m}^{3} / \mathrm{s}$ ).

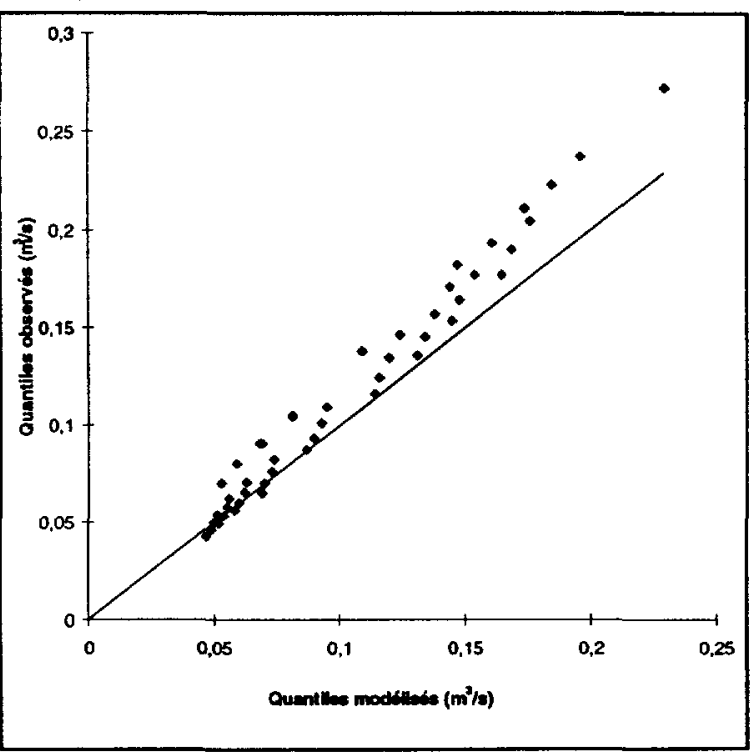

Figure 18 Ribita à RIBICIOARA « VCN $(T, d) \nsim$.

Ribita's basin at RIBICIOARA "VCN $(T, d)$ ".

$\left(\mathrm{De}=14,8 j\right.$ et $\left.\operatorname{VCN}(2,1)=0,087 \mathrm{~m}^{3} / \mathrm{s}\right)$ 


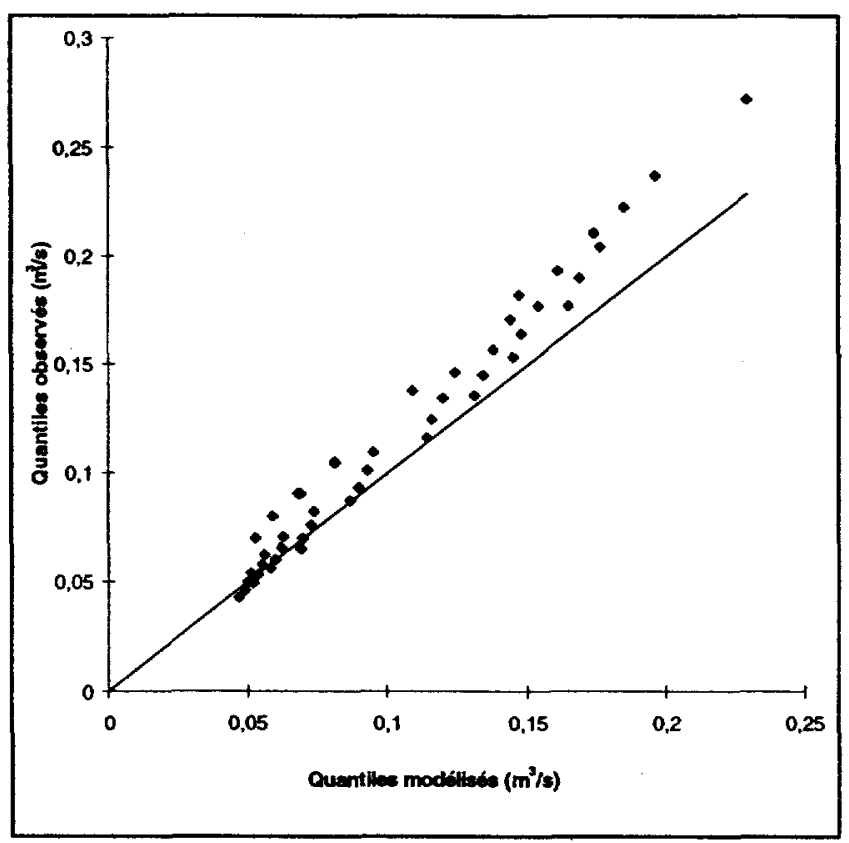

Figure 19 Crisu-Alb à CRISCIOR « VCN(T, d) ».

Crisu-Alb's basin at CRISCIOR "VCN $(T, d)$ ".

(De $=10,4$ jet $\left.V C N(2,1)=0,204 \mathrm{~m}^{3} / \mathrm{s}\right)$.

L'exemple de ces six bassins versants (figure 14 à 19) permet de montrer, qu'à partir de la connaissance des deux descripteurs locaux $(\operatorname{VCN}(2,1)$ et De) et du modèle $Q d F$ de référence (tableau 10), il est possible de typer le régime hydrologique d'étiage qui les caractérise. Les descripteurs locaux intègrent bien les effets d'échelle sur les processus d'écoulement de basses eaux relatifs : à l'aquifère (sol et formations sous-jacentes) de bassins versants de superficie $\mathrm{S}$ variable $\left(39 \mathrm{~km}^{2}\right.$ à $1581 \mathrm{~km}^{2}$, cf. tableau 10$)$, à T (2 ans à 50 ans, en année sèche ou humide) et à d ( 1 jà $30 j$ ).

\subsection{Résultats des modélisations en QCNd sur quelques sites}

De même que précédemment ( $\$ 6.2)$, nous montrons que les $\mathrm{QCN}(\mathrm{T}, \mathrm{d})$ modélisés sont cohérents avec ceux déduits des observations (figure 20 à 23). Pour le bassin du Banestilor à HALMAGIU, la durée d est limitée à 10 jours au lieu de 30 jours (hors des limites d'utilisation du modèle, cf. $§ 4.2$ ).

\subsection{Conclusion partielle}

Le bassin du Crisu-Alb en Roumanie est un premier exemple de validation qui confirme "et élargie " la représentativité spatiale (figure 11) et temporelle des modèles QdF de référence, mise en évidence sur le bassin hydrographique de la Loire. Nous rappelons, que pour chacun des bassins versants a été strictement appliqué le critère de choix des modèles QdF d'étiage établi précédemment 


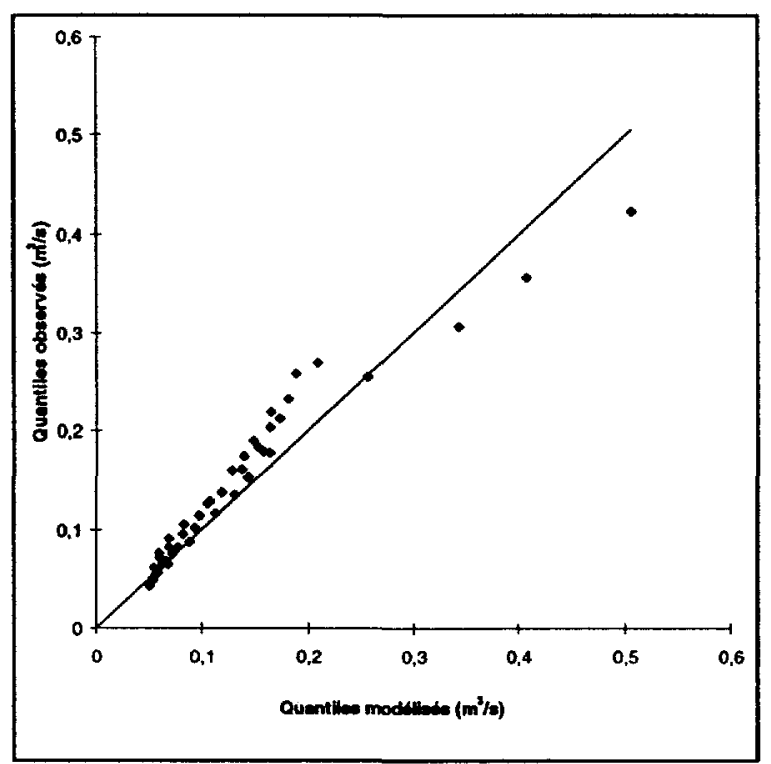

Figure 20 Ribita à RIBICIOARA * QCN(T, d) *.

Ribita's basin at RIBICIOARA "QCN(T, d)".

$\left(\mathrm{De}=14,8 \mathrm{j}\right.$ et $\left.\operatorname{VCN}(2,1)=0,087 \mathrm{~m}^{3} / \mathrm{s}\right)-\mathrm{Nash} \%: 89,2$.

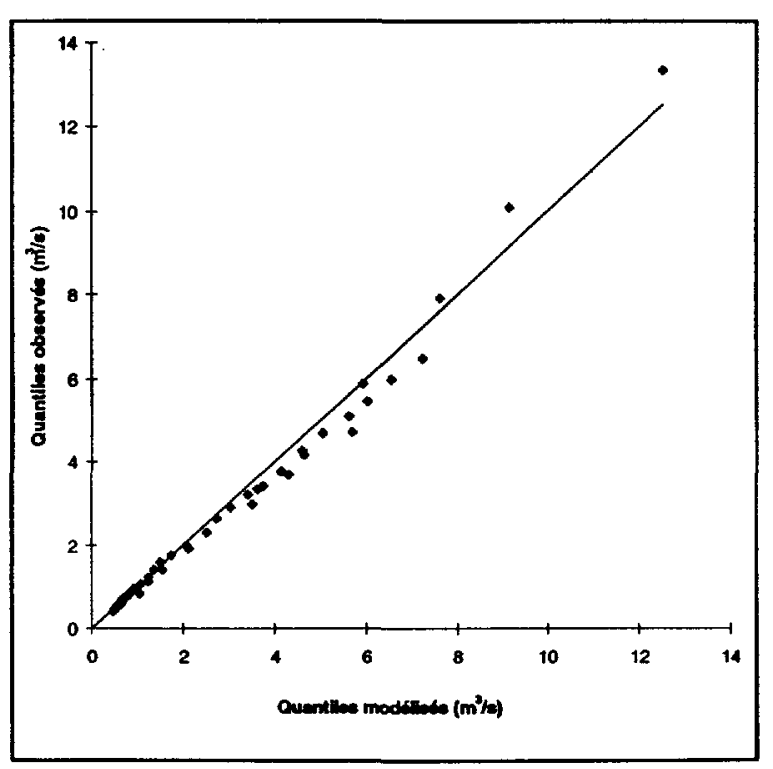

Figure 21 Crisu-Alb à GURAHONT “QCN(T, d) ».

Crisu-Alb's basin at GURAHONT "QCN $(T, d)$ ".

(De $=16,4$ jet $\left.\operatorname{VCN}(2,1)=1,419 \mathrm{~m}^{3} / \mathrm{s}\right)-\mathrm{Nash} \%: 98,3$. 


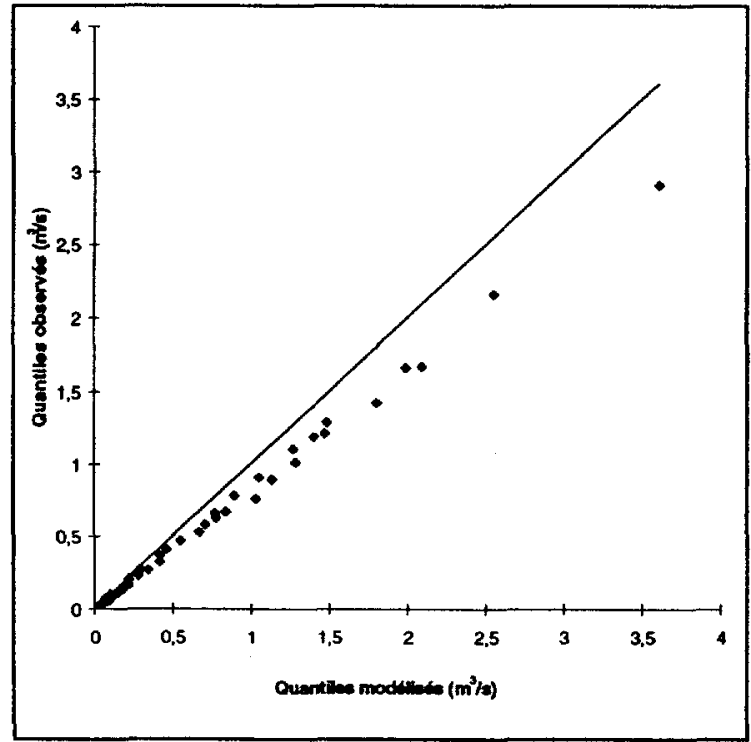

Figure 22 Crisu-Alb à CRISCIOR « QCN(T, d) ».

Crisu-Alb's basin at CRISCIOR "QCN $(T, d)$ ".

(De $=10,4$ jet $\left.\operatorname{VCN}(2,1)=0,204 \mathrm{~m}^{3} / \mathrm{s}\right)-\mathrm{Nash} \%: 90,9$.

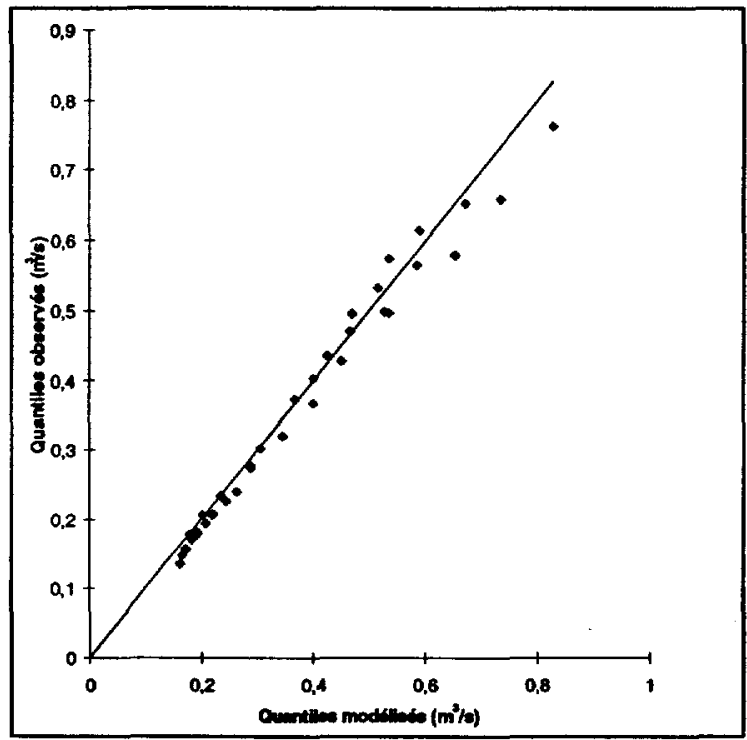

Figure 23 Banestilor à HALMAGIU « QCN(T, d) ».

Banestilor's basin at HALMAGIU "QCN $(T, d)$ ".

$\left(\mathrm{De}=8,8 \mathrm{j}\right.$ et $\left.\mathrm{VCN}(2,1)=0,278 \mathrm{~m}^{3} / \mathrm{s}\right)-\mathrm{Nash} \%: 97,5$. 
(§ 3.1). Ainsi, si nous associons le bassin du Crisu Alb à CRISCIOR à la famille hydrologique de VAUBARLET (écart-type 0,82 proche de 0,8 ) au lieu de FORCE, la modélisation en VCNd est moins performante, le Nash passe de $97,5 \%$ (tableau 10, figure 19) à $86,7 \%$ (figure 24). Pour ce qui concerne les QCNd, il y a un gain lié au valeurs humides fortes mieux modélisées, le Nash obtenu est de $95 \%$ (figure 25) au lieu de $90,9 \%$ (figure 22). Globalement, le modèle de FORCE donne une meilleure restitution des quantiles observés.

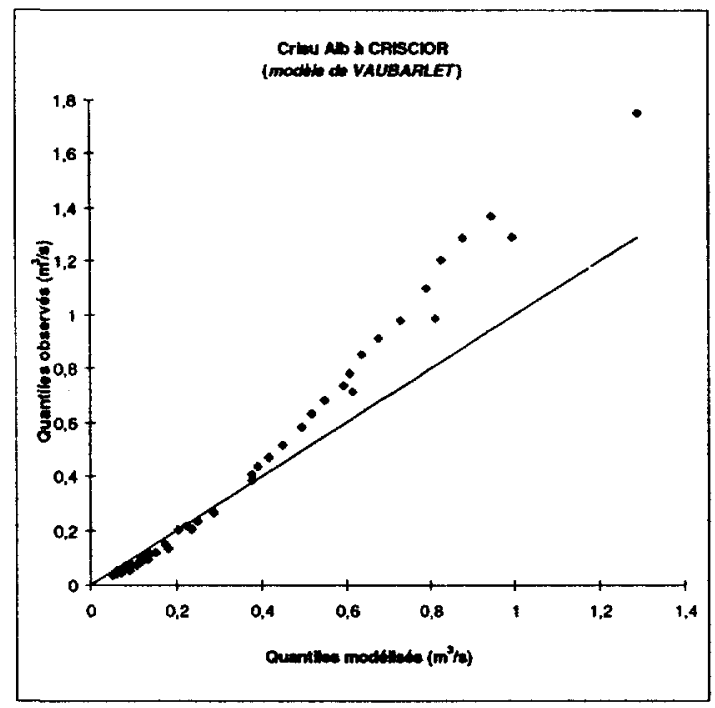

Figure 24 Quantiles VCN(T, d).

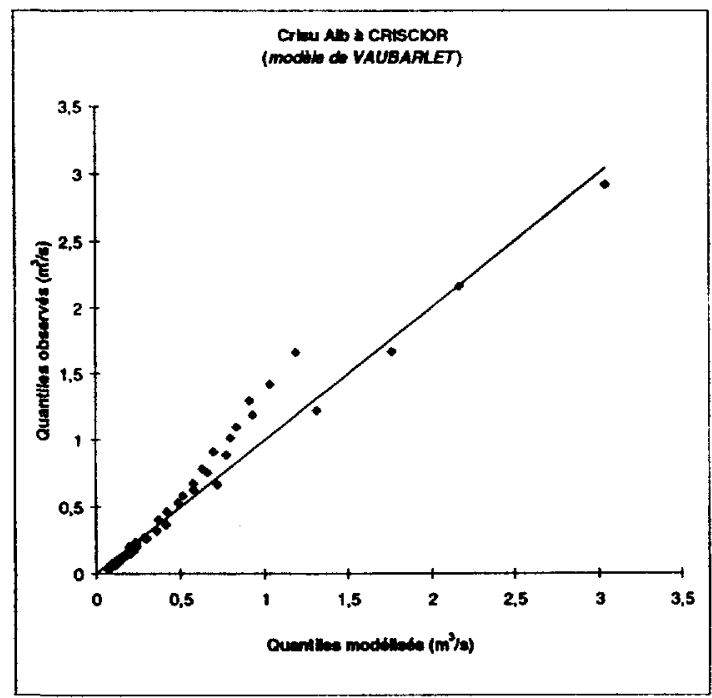

Figure 25 Quantiles QCN(T, d). 


\section{7 - SENSIBILITÉ À DE DES QUANTILES D'ÉTIAGE (EN VCNd) MODÉLISÉS}

Cette sensibilité peut être montrée sur l'exemple des bassins versants de l'Allier à MONISTROL D'ALLIER (figure 4) et de l'Alagnon à JOURSAC LE VIALARD (figure 26).

Pour chacun de ces deux bassins (ex. : tableau 8) sont connus les quantiles en VCNd observés et ceux estimés à partir des modèles QdF de référence, respectivement FORCE $\left(V C N(2,1)=0,845 \mathrm{~m}^{3} / \mathrm{s}\right.$ et $\left.D e=15,2 j\right)$ et SAINT-FLORET $\left(\operatorname{VCN}(2,1)=0,931 \mathrm{~m}^{3} / \mathrm{s}\right.$ et $\left.\mathrm{De}=17,9 \mathrm{j}\right)$. Les figures 27 et 28 permettent de voir comment l'erreur relative $(\%)$ entre quantiles observés et modélisés (année sèche ou humide) évolue avec De.

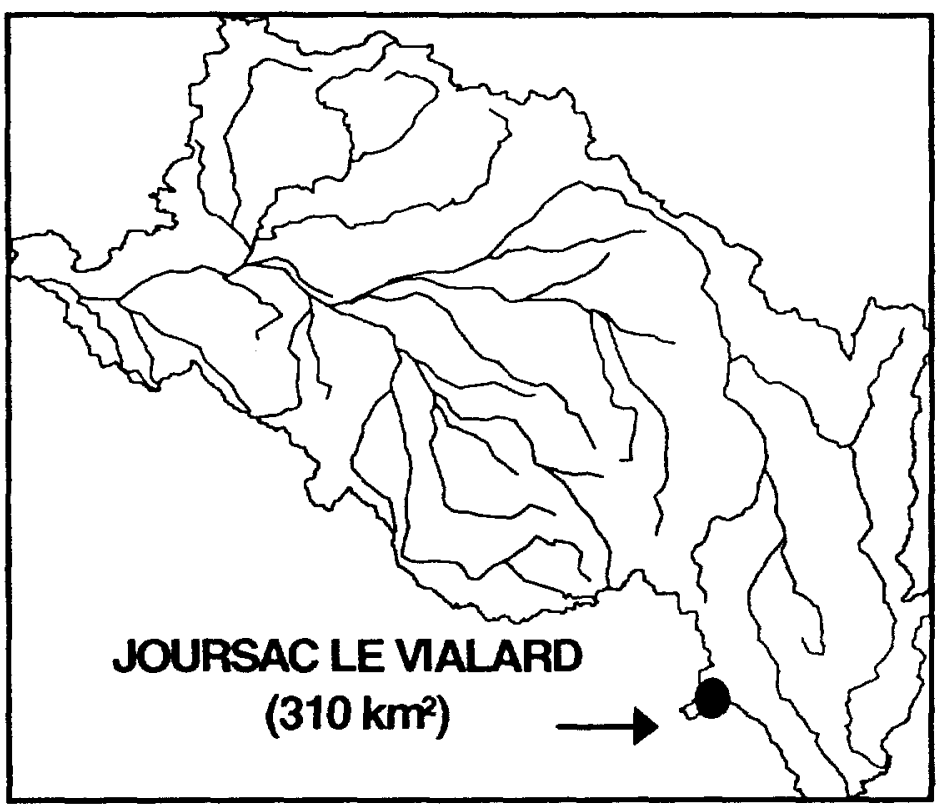

Figure 26 Localisation.

Location.

Les figures 27 et 28 montrent que la valeur de De estimée selon la médiane des $1 / \alpha_{i}$ (ex. : figure 5) est celle qui permet de minimiser l'erreur relative entre les quantiles observés et les quantiles modélisés. Autrement dit, elle permet d'avoir le meilleur compromis possible entre quantiles secs et quantiles humides modélisés, même si la qualité de la modélisation n'est pas homogène (figure 28) selon que l'on considère les quantiles en année sèche ou en année humide.

De manière générale, si De (médiane des $1 / \alpha_{\mathrm{i}}$ ) minimise bien les écarts entre quantiles observés et quantiles modélisés, on peut cependant admettre, suite aux résultats obtenus sur l'ensemble des sites étudiés du bassin de la Loire, une imprécision de l'ordre de $30 \%$ sur l'estimation de De sans remettre en cause la validité des résultats. 


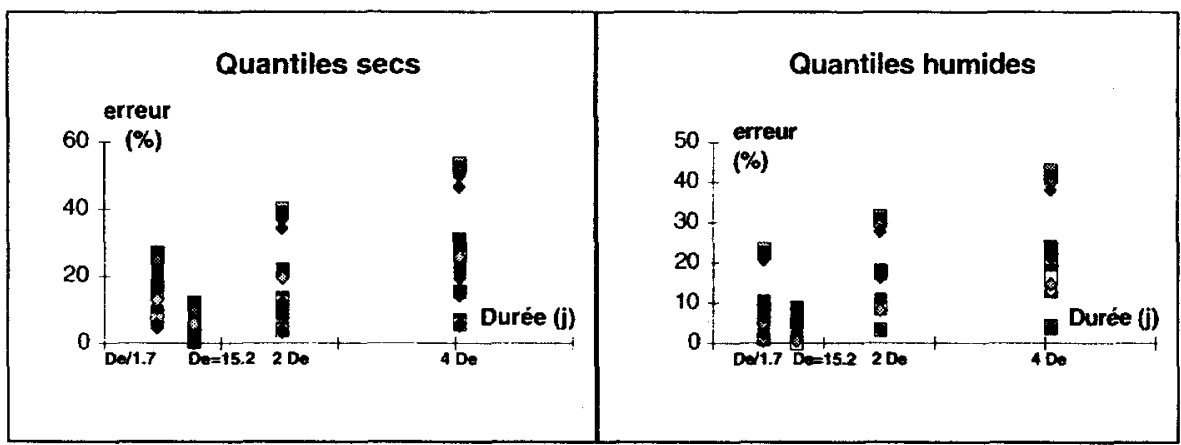

Figure 27 Bassin versant de l'Allier à MONISTROL d'ALLIER (S $=988 \mathrm{~km} 2$ ). Allier's basin at MONISTROL D'ALLIER (S $=988 \mathrm{~km} 2)$.

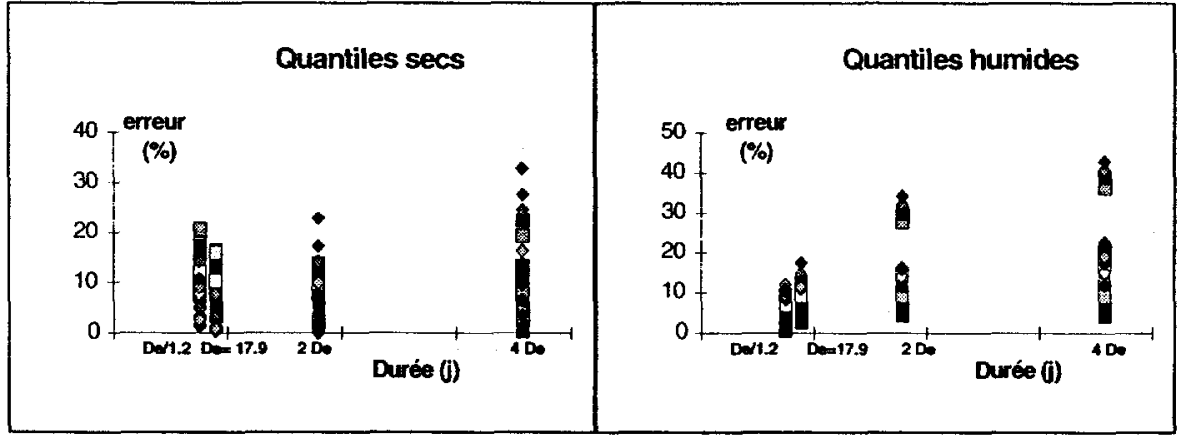

Figure 28 Bassin versant de l'Alagnon à JOURSAC LE VIALARD $\left(S=310 \mathrm{~km}^{2}\right)$. Alagnon's basin at JOURSAC LE VIALARD $\left(S=310 \mathrm{~km}^{2}\right)$.

\section{8 - RÉGIONALISATION DES RÉGIMES D'ÉTIAGE}

La régionalisation des régimes d'étiage doit permettre à priori l'usage des modèles QdF en des sites non observés. Pour cela, il était essentiel de montrer dans un premier temps, à partir des sites observés retenus sur les bassins hydrographiques de la Loire et du Crisu-Alb, la représentativité spatiale et temporelle (selon $T$ et $d$ ) des modèles QdF d'étiage de référence. Ensuite, pour permettre l'application de ces modèles en des sites non observés, une première tentative de régionalisation par krigeage des descripteurs locaux a été menée sur le bassin de la Loire (MERCIER et GALÉA, 1996). Comme l'on pouvait s'y attendre, les résultats obtenus sont peu fiables dans la mesure où (entre autres) les discontinuités de nature géologique, entre bassins versants, induisent un effet de pépite important. 


\section{CONCLUSION}

II nous a semblé essentiel de détailler, tant l'approche conceptuelle de la modélisation de synthèse des régimes de basses eaux que les premiers résultats obtenus (tableaux et graphiques), pour permettre une évaluation objective de la démarche. De manière générale, les biais induits par la modélisation (par rapport à la première bissectrice) ne sont pas systématiques et pas de même tendance. Par ailleurs, on peut considérer que les « biais » observés, en regard de la forte incertitude (non traitée) sur les quantiles d'étiage (erreur de mesure et d'échantillonnage sur les débits), ne sont pas de nature à compromettre les futurs développements sur cette thématique.

D'autres validations devront être effectuées pour renforcer la représentativité spatiale et temporelle (en $T$ et d) de ces modèles, en régime peu influencé et pérenne (quelques débits nuls acceptés), et affiner éventuellement les limites (écart-type, cf. § 3.1) différenciant les familles hydrologiques.

Le choix délibéré d'une loi de distribution simple $(L N)$, pour toute durée (d) et pour toute rivière, peut dans certains cas particuliers ne pas se vérifier (GUSTARD et al., 1989). II n'en demeure pas moins (notamment en régionalisation) que le fait de considérer un modèle simple multidurée contribue à un gain de cohérence de l'ensemble des résultats obtenus. En effet, il permet de valoriser toute l'information sur les basses eaux et de lisser d'une certaine manière l'erreur d'échantillonnage fréquente en étiage et ce d'autant plus que la durée considérée (d) est courte.

De manière générale, la méthodologie QdF développée en hydrologie synthétique des régimes hydrologiques d'étiage permet de bien rendre compte de la diversité des régimes observés. Au-delà de la loi de distribution choisie (LN), il était primordial de vérifier le concept même de la méthodologie QdF, autrement dit la force de liaison des descripteurs locaux avec les quantiles de différentes durées et fréquences (GUSTARD et al., 1989) des régimes de basses eaux (en année humide ou sèche).

Son aspect opérationnel, en matière de régionalisation, doit être poursuivi et développé. Notamment, l'estimation des paramètres d'entrée (ou descripteurs locaux du régime) des modèles $\mathrm{QdF}$ de référence nécessite des cartes de régionalisation des descripteurs de régime plus pertinentes que celles obtenues (MERCIER et GALÉA, 1996). Parallèlement au krigeage, qui perd de sa pertinence dans la mesure où les descripteurs n'ont pas vraiment de structure spatiale (même si l'on peut espérer une amélioration du variogramme par la prise en compte de la géologie), il devra être envisagé de corréler ces descripteurs avec d'autres variables physiographiques connues des bassins versants. De même, la méthode des jaugeages épisodiques (OBERLIN et al., 1973), qui semble donner des résultats intéressants (MERCIER et GALEA, 1996) quant à l'estimation du VCN(2, 1), devra être validée sur un plus grand nombre de bassins versants. Par ailleurs, l'usage opérationnel des modèles QdF d'étiage sur des sites non observés nécessitera, pour répondre aux objectifs de la loi sur l'eau de janvier 1993, d'expliciter le critère de choix (écart-type) d'appartenance du bassin étudié à l'une des quatre familles hydrologiques. 


\section{REMERCIEMENTS}

Nous remercions les services gestionnaires français et roumains pour leur collaboration, notamment en ce qui concerne la critique des observations de débit en général, et des débits de basses eaux, aux différents sites retenus.

\section{RÉFÉRENCES BIBLIOGRAPHIQUES}

Cemagref, CNRS, 1993. Approche écosystémique du bassin versant de la Loire : Éléments pour l'élaboration des orientations fondamentales de gestion. Phase I, État initial - Problématique. Atlas, Septembre.

CTGREF, 1979. Étude fréquentielle des étiages. Informations techniques $n^{\circ} 5$, cahier 33 .

GALÉA G., PRUDHOMME C., 1994. Modèles débit-durée-fréquence et conceptualisation d'un hydrogramme de crue synthétique: validation sur le BVRE de DRAIX, Hydrologie Continentale, 1994, vol. $9 n^{\circ} 2$.

GALÉA G., PRUDHOMME C., 1996. Notions de base et concepts utiles pour la compréhension de la modélisation synthétique des régimes de crue des bassins versants au sens des modèles QdF. Rev. Sci. Eau, vol. 10, $n^{\circ} 1,1997$, pp. 83-101.

GUSTARD A., ROALD L.A., DEMUTH $S$., LUMADJENG H.S., GROSS R., 1989. Regional low flow studies. FREND, Hydrol. Stud., vol. $n^{\circ} 1$, chap. 5, pp. 94-140.

KAABI A., 1994. Régionalisation des étiages dans le nord-ouest de l'Algérie. Thèse soutenue le 18/10/1994 devant l'école Doctorale de Mécanique (MMGE) de l'Université Joseph Fourier à Grenoble. GIS Hydrologie AMHY-France, Cemagref (Groupement de Lyon), ANRH (Alger).

LUBES H., MASSON J.M., RAOUS P., TAPIAU M., 1994. Safarhy, logiciel de calculs statistiques et d'analyse fréquentielle adapté à l'évaluation du risque en hydrologie-Manuel de référence, version 2.111. ORSTOM, Université Montpellier II.

MERCIER G.X., GALÉA G., 1996 : Transposition du concept $Q d F$ crue à la régionalisation des régimes d'étiage du bassin versant de la Loire (France) et du Crisu-Alb (Roumanie). Cemagref (groupement de Lyon), ENGEES (École Nationale du Génie de l'Eau ef de l'Environnement de Strasbourg).

OBERLIN G., GALÉA G., TONI J., 1973. Intérêt des jaugeages épisodiques pour l'estimation des étiages de petits bassins non équipés. Cahier ORSTOM, série hydrologie, vol. $X, n^{\circ} 4$, pp. 348-368.

OBERLIN G., LEDUC C., GAZULL L., 1989. Étiages en Languedoc-Roussillon. Cemagref (groupement de Lyon), Agence de Bassin Rhône-Méditerranée-Corse.

SOURISSEAU J., GALÉA G., 1996. Représentativité des modèles QdF, application à la régionalisation des régimes de crue du bassin versant de la Loire. Cemagref, groupement de Lyon - Division Hydrologie Hydraulique ; EPFL - IATE ; ministère de L'Environnement - Direction de l'Eau.

VOGEL R.M., KROLL C.N., 1991. The value of streamflow record augmentation procedures in low-flow and flood-flow frequency analysis. J. Hydrol., $n^{\circ} 125$, pp. 259-276. 